\title{
Flows between orthogonally stretching parallel plates
}

R. Ayats, ${ }^{1}$ F. Marques, ${ }^{1}$ A. Meseguer, ${ }^{1}$ and P. D. Weidman ${ }^{2}$

1)Department of Physics, Universitat Politècnica de Catalunya, Barcelona 08034, Spain

2)Department of Mechanical Engineering, University of Colorado, Boulder, CO 80309-0427, USA

(Dated: 29 December 2020)

Navier-Stokes equilibrium solutions of a viscous fluid confined between two infinite parallel plates that can independently stretch or shrink in orthogonal directions are studied. It is assumed that the admissible solutions satisfy spatial self-similarity in the stretching or shrinking perpendicular coordinates. The nonlinear steady boundary-value problem is discretized using a spectral Legendre method, and equilibrium solutions are found and tracked in the two-dimensional parameter space by means of pseudoarclength continuation Newton-Krylov schemes. Different families of solutions have been identified, some of which are two-dimensional and correspond to the classical Wang \& Wu self-similar flows arising in a plane channel with one stretching-shrinking wall; C. A. Wang and T.C. Wu, Comput. Math. Applic., 30, 1-16 (1995). However, a large variety of three-dimensional solutions have also been found, even for low stretching or shrinking rates. When slightly increasing those rates, some of these solutions disappear at saddle-node bifurcations. By contrast, when both plates are simultaneously stretching or shrinking at higher rates, a wide variety of new families of equilibria are created-annihilated in the neighbourhood of cuspidal codimension-2 bifurcation points. This behaviour has similarities with the one observed in other planar and cylindrical self-similar flows. 


\section{INTRODUCTION}

The study of viscous fluid motions bounded by stretching or shrinking surfaces is of fundamental importance in the mathematical modelling of physiological fluid flows (Waters, 2001, 2004). In these type of flows, it is usually assumed self-similarity of the Navier-Stokes solution, where the velocity field incorporates the spatial dependence of the non-uniform boundary conditions at the stretching or shrinking walls. Fluid motion induced by a single stretching flat plate was first studied by Crane (1970). The generalized problem, consisting of a biorthogonally stretching membrane was later addressed in Wang (1984) and more recently in Weidman and Ishak (2015). For a comprehensive review of exact Navier-Stokes solutions, including self-similar flow profiles see the monograph by Drazin and Riley (2006). For a recent update on these type of unbounded flows induced by extended stretching boundaries, see the more recent review by Wang (2011). In Crane's original formulation, as well as in its biorthogonal generalization, it is assumed that the wall-normal coordinate is unbounded. By contrast, other studies have explored flows arising in two-dimensional channels with confining parallel walls stretching and shrinking in the streamwise direction (Brady and Acrivos, 1981; Marques et al., 2017). In these planar geometries, alternative finite difference discretizations providing more realistic boundary conditions have been recently proposed (Espín and Papageorgiou, 2009). Although these formulations do not impose the selfsimilarity ansatz in the interior of the computational domain, the observed dynamics are consistent with the extensional computations, at least for moderate Reynolds numbers.

In this work, we address the generalization of extensional flows in planar channels whose confining parallel walls are biorthogonally and independently stretching or shrinking at arbitrary acceleration rates. In particular, we search for equilibrium solutions (steady flows) which are exact solutions of the Navier-Stokes equations under the assumption of the self-similar ansatz. We aim to provide a comprehensive and detailed description of possible equilibria by means a highly accurate Legendre spectral spatial discretisation of the Navier-Stokes self-similar boundary value problem, followed by a robust pseudoarclength Newton-Krylov continuation method, capable of tracking all solutions and their potential bifurcations. The use of spectral methods is particularly convenient in this case due to the simplicity of the geometry and boundary conditions, allowing for an exponential (or spectral) convergence of the discretized solutions.

The paper is structured as follows. Section $\S I I$ is devoted to the mathematical formulation of the problem, where the self-similar ansatz is introduced and the governing system of nonlinear partial 
(a)

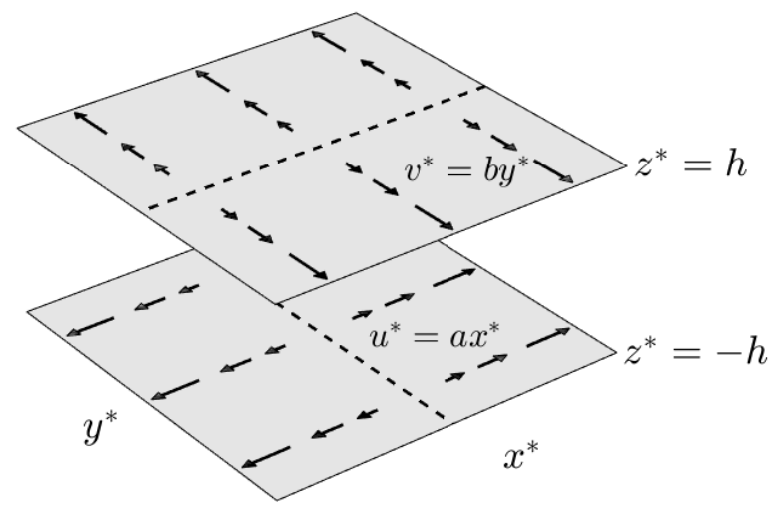

(b)

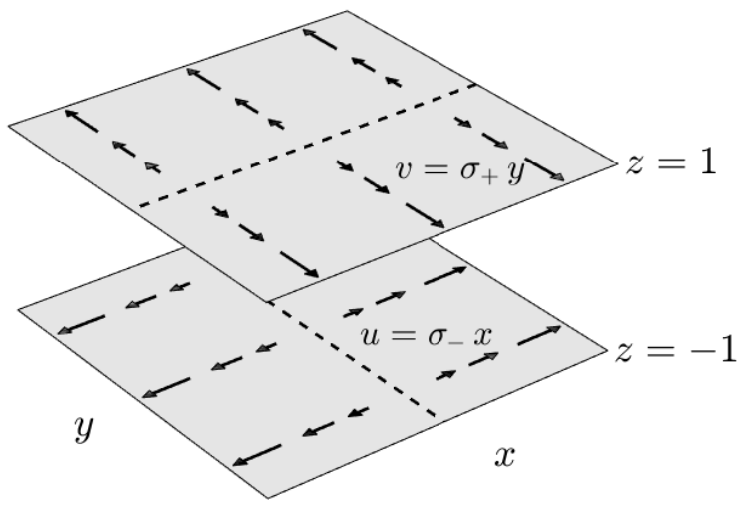

FIG. 1. Schematics of the problem. (a) In dimensional form. (b) Using non-dimensional variables.

differential equations are obtained. Section $\S I I$ also introduces the symmetry transformations that leave invariant the domain, the boundary conditions, and the self-similarity of the solution. The spectral Legendre spatial discretization of the aforementioned partial differential equations is described in detail in Section §III. Section $§ I V$ is focused on the search of steady flow solutions for moderate stretching-shrinking rates of the plates. Section $\S \mathrm{V}$ describes the mechanism by which new and old solutions are created and annihilated when the stretching-shrinking rates are increased and, in particular, the role of codimension-2 cusp bifurcations in such mechanism. Finally, Section $\S$ VI summarizes the main findings of the current exploration.

\section{MATHEMATICAL FORMULATION}

We consider a viscous fluid of kinematic viscosity $v$ and density $\rho$ confined between two infinite parallel plates separated by a distance $2 h$, as depicted in Fig. 1(a). The problem is formulated in Cartesian coordinates $\left(x^{*}, y^{*}, z^{*}\right)$, where starred variables henceforth stand for dimensional quantities. In this coordinate system, the motion of the fluid between the parallel plates is described by the velocity field $\mathbf{u}^{*}=\left(u^{*}, v^{*}, w^{*}\right)$. As shown in Fig. 1(a), the lower and upper plates are located at the wall-normal coordinates $z^{*}= \pm h$, linearly stretching along the $x^{*}$ and $y^{*}$ coordinates, respectively. The velocities of the fluid on the lower and upper impermeable plates are

$$
\begin{array}{ccc}
u^{*}\left(x^{*}, y^{*},-h\right)=a x^{*}, & v^{*}\left(x^{*}, y^{*},-h\right)=0, & w^{*}\left(x^{*}, y^{*},-h\right)=0, \\
u^{*}\left(x^{*}, y^{*}, h\right)=0, & v^{*}\left(x^{*}, y^{*}, h\right)=b y^{*}, & w^{*}\left(x^{*}, y^{*}, h\right)=0
\end{array}
$$


where $a$ and $b$ are the strain rate of stretching along the $x^{*}$ and $y^{*}$-axis respectively.

The problem is rendered nondimensional after using $h, h^{2} / v, v / h$ and $\rho v^{2} / h^{2}$ as units of length, time, velocity, and pressure, respectively, leading to the dimensionless incompressible Navier-Stokes equations

$$
\nabla \cdot \mathbf{u}=0, \quad \partial_{t} \mathbf{u}+(\mathbf{u} \cdot \nabla) \mathbf{u}=-\nabla p+\nabla^{2} \mathbf{u}
$$

In dimensionless coordinates, the boundary conditions read

$$
\begin{gathered}
u(x, y,-1)=\frac{a h^{2}}{v} x \equiv \sigma_{-} x, \quad v(x, y,-1)=0, \quad w(x, y,-1)=0, \\
u(x, y, 1)=0, \quad v(x, y, 1)=\frac{b h^{2}}{v} y \equiv \sigma_{+} y, \quad w(x, y, 1)=0,
\end{gathered}
$$

where the new dimensionless quantities $\sigma_{-}=a h^{2} v^{-1}$ and $\sigma_{+}=b h^{2} v^{-1}$ introduced in (4) and (5) measure the strain rates of the lower and upper plates, respectively. In what follows, we assume the solenoidal self-similar ansatz

$$
u(x, y, z, t)=x f^{\prime}(z, t), v(x, y, z, t)=y g^{\prime}(z, t), w(x, y, z, t)=-(f+g)
$$

Formal substitution of (6) into the incompressible Navier-Stokes equations (3) yields the nonlinearly coupled relations

$$
\begin{gathered}
f^{\prime \prime \prime}+(f+g) f^{\prime \prime}-f^{2}-\partial_{t} f^{\prime}=\beta_{1}, \\
g^{\prime \prime \prime}+(f+g) g^{\prime \prime}-g^{2}-\partial_{t} g^{\prime}=\beta_{2},
\end{gathered}
$$

where a prime denotes differentiation with respect to the nondimensional wall-normal coordinate $z$, and $\beta_{1}$ and $\beta_{2}$ are constants to be determined. The corresponding pressure distribution is

$$
p(x, y, z, t)=\frac{1}{2}\left(\beta_{1} x^{2}+\beta_{2} y^{2}\right)-f^{\prime}-g^{\prime}-\frac{1}{2}(f+g)^{2}+\partial_{t} \int(f+g) d z .
$$

Assuming zero mass-flux along finite vertical planes parallel to the $x$ and $y$ axes, the solutions $f$ and $g$ of system (7) satisfy the boundary conditions

$$
\begin{aligned}
& f^{\prime}(-1)=\sigma_{-}, \quad f(-1)=0, \quad f(1)=0, \quad f^{\prime}(1)=0 \\
& g^{\prime}(-1)=0, \quad g(-1)=0, \quad g(1)=0, \quad g^{\prime}(1)=\sigma_{+} .
\end{aligned}
$$

Following Marques et al. (2017), we introduce the two alternative parameters

$$
\sigma_{+}=R \cos \alpha, \quad \sigma_{-}=R \sin \alpha
$$

where $R=\sqrt{\sigma_{+}^{2}+\sigma_{-}^{2}}$ is the Reynolds number, and $0 \leq \alpha \leq 2 \pi$ measures the relative strength of the two streching walls. This gives rise to four regions 
Orthogonally stretching parallel plates

Region 1. $0 \leq \alpha \leq \pi / 2 \quad$ (top stretches, bottom stretches)

Region 2. $\pi / 2 \leq \alpha \leq \pi \quad$ (top shrinks, bottom stretches)

Region 3. $\pi \leq \alpha \leq 3 \pi / 2$ (top shrinks, bottom shrinks)

Region $4.3 \pi / 2 \leq \alpha \leq 2 \pi$ (top stretches, bottom shrinks)

For purposes of integration we take the derivative of (7) to obtain the $8^{\text {th }}$ order system

$$
\begin{gathered}
\partial_{t} f^{\prime \prime}=f^{i v}+(f+g) f^{\prime \prime \prime}-\left(f^{\prime}-g^{\prime}\right) f^{\prime \prime} \\
\partial_{t} g^{\prime \prime}=g^{i v}+(f+g) g^{\prime \prime \prime}+\left(f^{\prime}-g^{\prime}\right) g^{\prime \prime}
\end{gathered}
$$

and calculate the pressure gradients from any of the relevant equations as

$$
\beta_{1}=f^{\prime \prime \prime}(1)=f^{\prime \prime \prime}(-1)-1, \quad \beta_{2}=g^{\prime \prime \prime}(-1)=g^{\prime \prime \prime}(1)-1
$$

Relations for the $x$ and $y$ directed wall shear stresses at the upper and lower walls are given in nondimensional form (unit $\rho v^{2} / h^{2}$ ) as

$$
\tau_{x}=\tau_{x z}=x f^{\prime \prime}( \pm 1), \quad \tau_{y}=\tau_{y z}=y g^{\prime \prime}( \pm 1)
$$

We have used the dimensional expression

$$
\tau_{i j}^{*}=\mu\left(\partial_{i} u_{j}+\partial_{j} u_{i}\right)^{*}=\frac{\rho v^{2}}{h^{2}}\left(\partial_{i} u_{j}+\partial_{j} u_{i}\right)=\frac{\rho v^{2}}{h^{2}} \tau_{i j}
$$

Assuming the flow is steady, and neglecting the nonlinear terms appearing in (11), the solution must satisfy $f^{i v}=g^{i v}=0$. In this case we obtain the third order polynomials

$$
\begin{aligned}
& f_{0}(z)=\frac{\sigma_{-}}{4}(z-1)^{2}(z+1)=\frac{R \sin \alpha}{4}\left(z^{3}-z^{2}-z+1\right) \\
& g_{0}(z)=\frac{\sigma_{+}}{4}(z+1)^{2}(z-1)=\frac{R \cos \alpha}{4}\left(z^{3}+z^{2}-z-1\right)
\end{aligned}
$$

that lead to the base Stokes flow

$$
\begin{gathered}
u_{0}(x, z)=x \frac{R \sin \alpha}{4}\left(3 z^{2}-2 z-1\right), \quad v_{0}(y, z)=y \frac{R \cos \alpha}{4}\left(3 z^{2}+2 z-1\right) \\
w_{0}(x, y, z)=\frac{R}{4}\left(1-z^{2}\right)[\sin \alpha(z-1)+\cos \alpha(z+1)]
\end{gathered}
$$

depicted in Fig. 2 for $R=8$ and selected values of $\alpha$. This base flow is not a solution of the Navier-Stokes equations and the advection term modifies the flow, as can be seen by comparing the base flow in Fig. 2 with the steady solution $A_{1}$ in Fig. 5, which is the self-similar solution of 

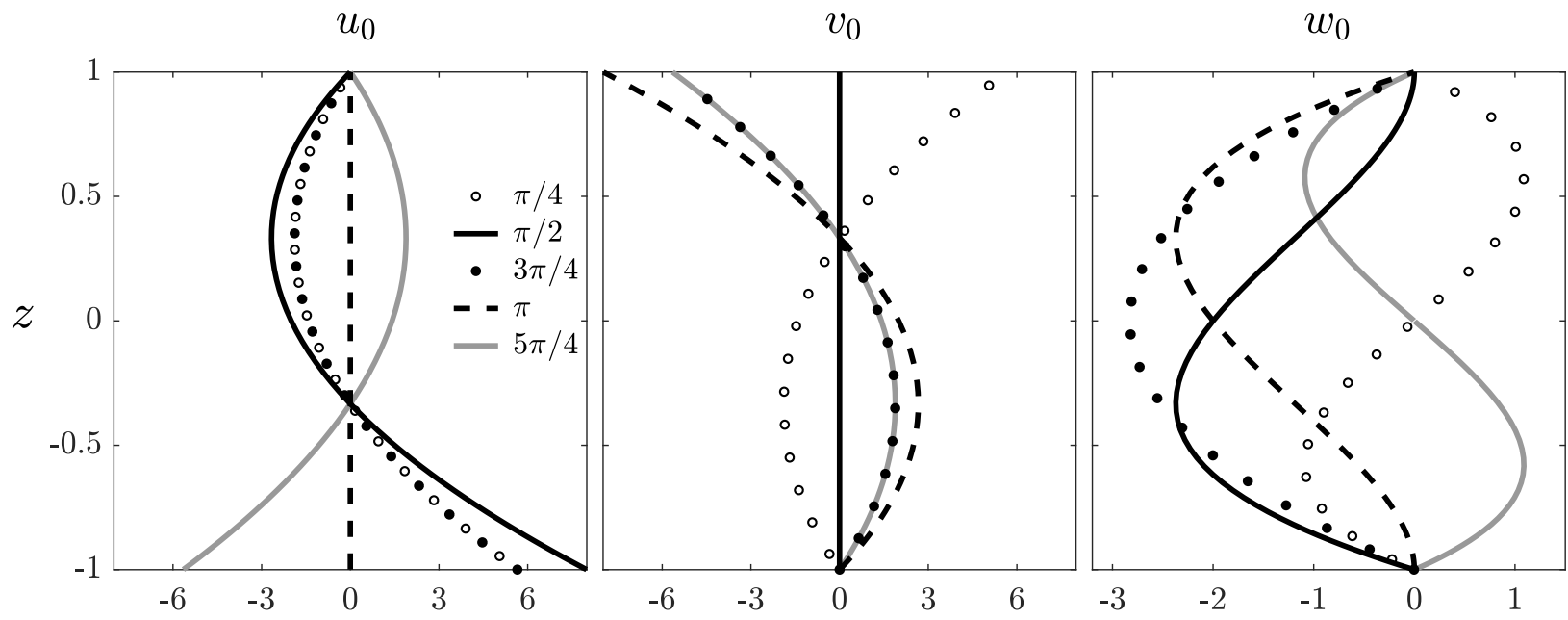

FIG. 2. Structure of the base Stokes flow (16) at $R=8$ for the $\alpha$ values indicated. Panels show velocity profiles of $u_{0}, v_{0}$ and $w_{0}$ of (16) at $x=1$ and $y=1$.

the Navier-Stokes equations at $R=8$ that smoothly connects with the base flow for $R \rightarrow 0$. This connection can be clearly seen in Fig. 5, where the $U$ and $V$ components of the $\mathrm{A}_{1}$ solution are very similar to the Stokesian profile $u_{0}$ and $v_{0}$, respectively, shown in Fig. 2. However, the effects of the advection already lead to noticeable differences in the wall-normal components $W$ and $w_{0}$, particularly for $\alpha=\pi$ and $\alpha=\pi / 2$.

\section{A. Symmetries}

The domain, along with the governing equations (11), the boundary conditions (9), and the self-similar ansatz (6), are invariant with respect to the two specular reflections about the planes $x=0$ and $y=0$

$$
K_{x}:(x, y, z) \mapsto(-x, y, z), \quad K_{y}:(x, y, z) \mapsto(x,-y, z)
$$

The domain and governing equations (but not the boundary conditions) satisfy the additional reflectional symmetry about the mid plane

$$
K_{z}:(x, y, z) \mapsto(x, y,-z)
$$

In order to get the correct direction of the velocities on the domain boundary, we can compose this reflection with a reflection about the plane bisector of the first quadrant, $x=y$, resulting in the 
Orthogonally stretching parallel plates

rotation of $\pi$ around the straight line $x=y, z=0$ :

$$
H:(x, y, z) \mapsto(y, x,-z)
$$

This symmetry operation leaves invariant the domain and governing equations, and exchanges $\sigma_{+}$ and $\sigma_{-}$(or what is the same, changes $\alpha$ into $\pi / 2-\alpha$ ). It is therefore a true symmetry of the problem for the particular cases $\sigma_{+}=\sigma_{-}$, i.e. for $\alpha=\pi / 4$ and $5 \pi / 4$.

According to the previous considerations, the induced action of the symmetries on the velocity field are

$$
\begin{aligned}
& K_{x}:[u, v, w](x, y, z, t ; R, \alpha) \mapsto[-u, v, w](-x, y, z, t ; R, \alpha), \\
& K_{y}:[u, v, w](x, y, z, t ; R, \alpha) \mapsto[u,-v, w](x,-y, z, t ; R, \alpha), \\
& H:[u, v, w](x, y, z, t ; R, \alpha) \mapsto[v, u,-w](y, x,-z, t ; R, \pi / 2-\alpha) .
\end{aligned}
$$

The self-similar anzatz (6) is left invariant by $K_{x}$ and $K_{y}: K_{x} \mathbf{u}=K_{y} \mathbf{u}=\mathbf{u}$. The only non-trivial action that remains in the self-similar formulation is the half-turn $H$, whose square is the identity. The action of $H$ on $f$ and $g$ is

$$
H:[f, g](z, t ; R, \alpha) \mapsto[-g,-f](-z, t ; R, \pi / 2-\alpha)
$$

As a consequence of the $H$-symmetry, from any solution for $(R, \alpha)$, another solution for $(R, \pi / 2-$ $\alpha)$ is obtained. Therefore, it is sufficient to compute the solutions for $\alpha \in[\pi / 4,5 \pi / 4]$, and then apply the $H$ symmetry to obtain the solutions in the whole interval $\alpha \in[0,2 \pi]$.

It is convenient to represent the velocity field of a given solution by plotting the $z$-profiles $\mathbf{u}(1,1, z, t)$ :

$$
\mathbf{u}(1,1, z, t)=(U(z, t), V(z, t), W(z, t))=\left(f^{\prime}, g^{\prime},-f-g\right) .
$$

From them the whole velocity field at any $(x, y)$ value is obtained by using (6). The action of $H$ on $(U, V, W)$ is

$$
H:[U, V, W](z, t ; R, \alpha) \mapsto[V, U,-W](-z, t ; R, \pi / 2-\alpha) .
$$

Another useful feature of the flow is its helicity, defined as

$$
h=\mathbf{u} \cdot(\nabla \times \mathbf{u})
$$

that is a pseudo-scalar (i. e., it changes sign under reflections $K_{x}$ and $K_{y}$ ), and it is a true scalar for rotations $H$. For a self-similar solution, the expression of $h$ is

$$
h(x, y, z, t)=x y\left(g^{\prime} f^{\prime \prime}-f^{\prime} g^{\prime \prime}\right) .
$$


Orthogonally stretching parallel plates

Its symmetry properties are

$$
\begin{gathered}
h(x, y, z, t ; R, \alpha)=-h(-x, y, z, t ; R, \alpha)=-h(x,-y, z, t ; R, \alpha), \\
H: h(x, y, z, t ; R, \alpha) \mapsto h(y, x,-z, t ; R, \pi / 2-\alpha) .
\end{gathered}
$$

\section{SPECTRAL LEGENDRE DISCRETIZATION}

Henceforth in this study we seek steady solutions of (11), satisfying

$$
\begin{aligned}
& f^{i v}+(f+g) f^{\prime \prime \prime}-\left(f^{\prime}-g^{\prime}\right) f^{\prime \prime}=0, \\
& g^{i v}+(f+g) g^{\prime \prime \prime}+\left(f^{\prime}-g^{\prime}\right) g^{\prime \prime}=0
\end{aligned}
$$

where $f(z)$ and $g(z)$ are functions defined within the domain $z \in[-1,1]$, that satisfy the boundary conditions

$$
f(-1)=0, f^{\prime}(-1)=R \sin \alpha, f(1)=0, f^{\prime}(1)=0,
$$

and

$$
g(-1)=0, g^{\prime}(-1)=0, g(1)=0, g^{\prime}(1)=R \cos \alpha
$$

To simplify the numerical setting, we consider the splittings

$$
f(z)=f_{0}(z)+F(z), g(z)=g_{0}(z)+G(z)
$$

where $f_{0}(z)$ and $g_{0}(z)$ constitute the basic Stokes solution (15) satisfying the boundary conditions (28) and (29), and $F(z)$ and $G(z)$ are deviations from the basic flow satisfying Dirichlet-Neumann homogeneous boundary conditions

$$
F( \pm 1)=G( \pm 1)=F^{\prime}( \pm 1)=G^{\prime}( \pm 1)=0 .
$$

After introducing (30) in (27), the BVP reads

$$
\begin{aligned}
& F^{i v}+\left(f_{0}+F+g_{0}+G\right)\left(f_{0}^{\prime \prime \prime}+F^{\prime \prime \prime}\right)-\left(f_{0}^{\prime}+F^{\prime}-g_{0}^{\prime}-G^{\prime}\right)\left(f_{0}^{\prime \prime}+F^{\prime \prime}\right)=0 \\
& G^{i v}+\left(f_{0}+F+g_{0}+G\right)\left(g_{0}^{\prime \prime \prime}+G^{\prime \prime \prime}\right)+\left(f_{0}^{\prime}+F^{\prime}-g_{0}^{\prime}-G^{\prime}\right)\left(g_{0}^{\prime \prime}+G^{\prime \prime}\right)=0
\end{aligned}
$$

or simply

$$
\mathbf{N}\left(F, G, f_{0}, g_{0}\right)=\left[\begin{array}{l}
N_{1}\left(F, G, f_{0}, g_{0}\right) \\
N_{2}\left(F, G, f_{0}, g_{0}\right)
\end{array}\right]=\left[\begin{array}{l}
0 \\
0
\end{array}\right],
$$


where $N_{1}$ and $N_{2}$ stand for nonlinear differential operators acting on the sought functions $F$ and $G$, implicitly depending on the Stokes base fields $f_{0}$ and $g_{0}$. We discretize the nonlinear differential system (32) using spectral expansions of the form

$$
\left[\begin{array}{l}
F(z) \\
G(z)
\end{array}\right]=\sum_{m=0}^{M} a_{m} \Phi_{m}^{(1)}(z)+b_{m} \Phi_{m}^{(2)}(z)
$$

where

$$
\Phi_{m}^{(1)}(z)=\left[\begin{array}{c}
\phi_{m}(z) \\
0
\end{array}\right], \Phi_{m}^{(2)}(z)=\left[\begin{array}{c}
0 \\
\phi_{m}(z)
\end{array}\right], \phi_{m}(z)=\left(1-z^{2}\right)^{2} P_{m}(z),
$$

and where $P_{m}(z)$ is the $m$ th Legendre polynomial. We introduce the inner Hermitian product between two arbitrary vector fields $\Psi(z)=\left[\psi_{1}(z) \psi_{2}(z)\right]^{\mathrm{T}}$ and $\Theta(z)=\left[\theta_{1}(z) \theta_{2}(z)\right]^{\mathrm{T}}$

$$
(\Psi, \Phi) \doteq \int_{-1}^{1} \Psi(z) \cdot \Theta(z) \mathrm{d} z
$$

The Galerkin formulation consists in formally substituting the spectral expansion (34) in (33) and projecting on the set of quasi-orthogonal vector fields $\Phi_{m}^{(1)}$ and $\Phi_{m}^{(2)}$ defined in (35). These Hermitian products are computed with a Gauss-Legendre quadrature, which provides a maximum exactness with a reduced number of nodes (Quarteroni, Sacco, and Saleri, 2010), and the spectral expansion is evaluated on the corresponding nodes so that the wall-normal integral can be accurately computed. This results in an algebraic system of nonlinear equations for the spectral coefficients $\mathbf{a}=\left[a_{0} a_{1} \cdots a_{M}\right]$ and $\mathbf{b}=\left[b_{0} b_{1} \cdots b_{M}\right]$, that is:

$$
\begin{aligned}
& \left(\Phi_{m}^{(1)}, \mathbf{N}\left(F, G, f_{0}, g_{0}\right)\right)=\mathscr{N}_{1}(\mathbf{a}, \mathbf{b}, R, \alpha)=0 \\
& \left(\Phi_{m}^{(2)}, \mathbf{N}\left(F, G, f_{0}, g_{0}\right)\right)=\mathscr{N}_{2}(\mathbf{a}, \mathbf{b}, R, \alpha)=0 .
\end{aligned}
$$

The above system defines $\mathbf{a}$ and $\mathbf{b}$ as implicit functions of $R$ and $\alpha$. All derivatives in the wallnormal direction have been computed by means of differentiation matrices based on Legendre nodes (Meseguer, 2020). System (37) is solved using matrix-free Newton-Krylov methods (Kelley, 2003) that only require the action of the Jacobian operator instead of the full matrix typically required by classical Newton-Raphson methods. The numerical solution of system (37) is particularly challenging in the neighbourhood of folds or cuspidal points, where the Jacobian is extremely ill-conditioned. All the solutions of system (37) reported henceforth in the present work are computed using $M=50$ Legendre polynomials and converged to the tolerance condition $\sqrt{\mathscr{N}_{1}^{2}+\mathscr{N}_{2}^{2}}<10^{-12}$, which ensures reliable results for all bifurcations and singular points, as 
Appendix A illustrates. The solutions found have been subsequently tracked (homotopic transformations) in the two-dimensional $(\alpha, R)$ parameter space by means of pseudoarclength continuation methods Kuznetsov (2004). It is worth mentioning that these methods are specially challenging when a branch approaches a critical point, like cusps or folds, as the Jacobian matrix is ill-conditioned near to singular points. The homotopic methods employed in the present work, along with complementary methodologies to identify saddle-node (fold) bifurcations or cusp points, have been previously used successfully for the continuation of equilibria in extensional planar and cylindrical geometries (Marques et al., 2017; Marques and Meseguer, 2019) and also in more generic shear or annular hydrodynamic and hydromagnetic flows (Meseguer et al., 2009; Deguchi, Meseguer, and Mellibovsky, 2014; Ayats et al., 2020; Ayats, Meseguer, and Mellibovsky, 2020).

\section{FAMILIES OF STEADY SOLUTIONS FOR LOW-MODERATE $R$}

We start the exploration of steady solutions by reproducing the results of a former analysis carried out in Wang and Wu (1995) for a two-dimensional channel, where one of the plates stretches at a constant rate and the other remains stationary. In our formulation, Wang \& Wu's problem can therefore be recovered by setting in system (37) $\alpha=0$ (top plate stretching in the $y$-direction and lower plate stationary), or $\alpha=\pi / 2$ (lower plate stretching in the $x$-direction and upper plate stationary). Figure 3 shows the continuation curves of all identified branches for $\alpha=0$ as a function of the Reynolds number within the range $R \in[-20,110]$. One of these branches, namely $\mathrm{A}_{1}$ (black curves in Fig. 3), is the solution branch found in Wang and Wu (1995). For consistency, Fig. 3(a) also includes an inset plot depicting Wang \& Wu's solution in terms of the normalized pressure factor $\beta=-\beta_{2} / R$ used in their analysis, showing a perfect agreement with the zoom in of branches II and III detailed in Figure 2 of Wang and Wu (1995). The analysis carried out in Wang \& Wu also studied the flow for negative acceleration (shrinking) of one of the plates, that is for $R<0$. In our computations, branch $\mathrm{A}_{1}$ has also been continued for negative values of the Reynolds number. In particular, Fig. 3(a) shows branch $A_{1}$ intercepting the ordinate $\beta_{2}=334.4$ for $R=-20$, leading to a pressure factor $\beta=-16.7$ that is in very good agreement with the value of $\beta$ showed in Figure 2 of Wang and Wu (1995).

The other branches appearing in Fig. 3 have been found after performing a comprehensive exploration of solutions of system (37) for different values of the parameter $\alpha$ and tracking them 
(a)

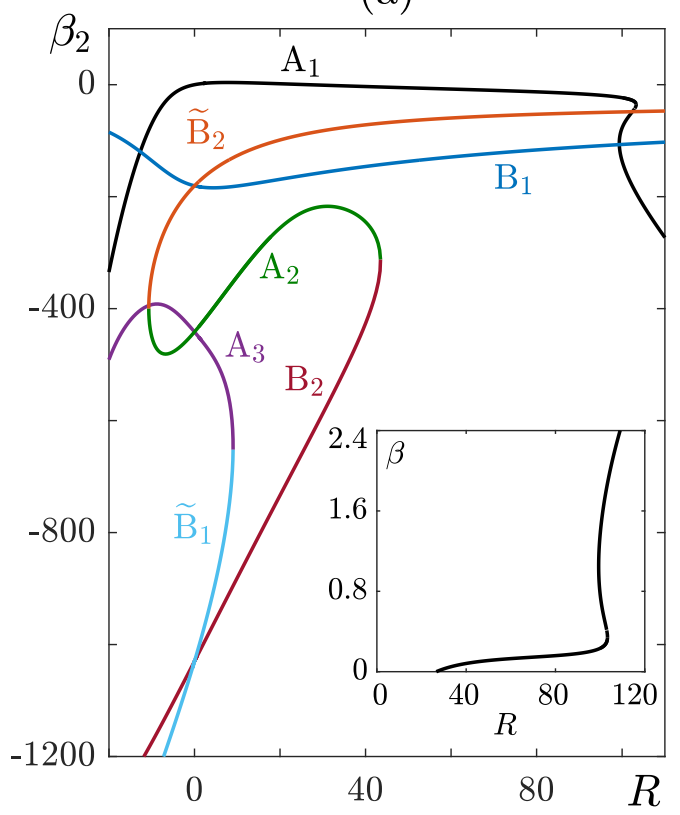

(b)

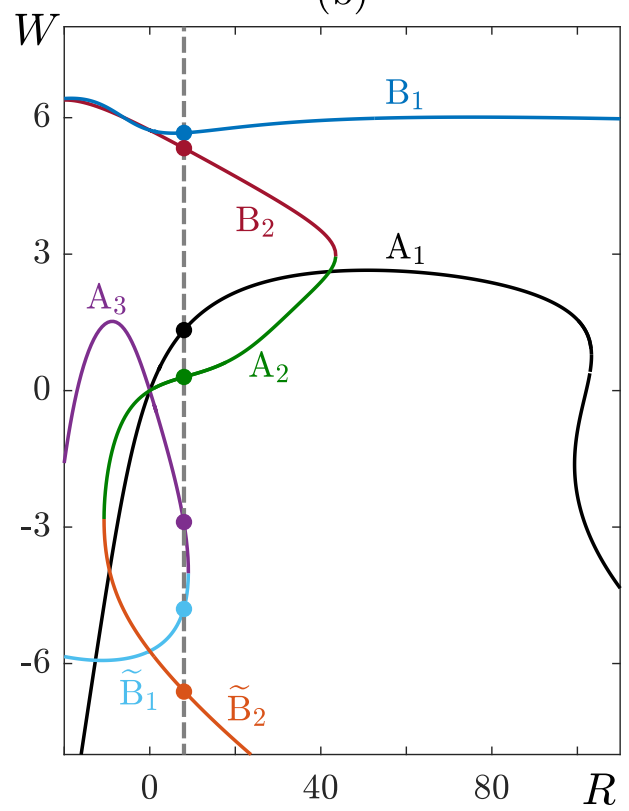

FIG. 3. Continuation of solution branches for $\alpha=0$. In black, Wang \& Wu's $\left(\mathrm{A}_{1}\right)$ solution branch formerly reported in Wang and $\mathrm{Wu}$ (1995). (a) Pressure factor $\beta_{2}$ (the inset shows the normalized factor $\beta=-\beta_{2} / R$, following Figure 2 of Wang and $\mathrm{Wu}(1995))$. (b) Wall-normal velocity $W$ at mid-plane $z=0$. A gray dashed vertical line has been placed at $R=8$, along with color coded bullets, to guide the eye in the $\alpha$-unfolding of the solution branches, depicted in Fig. 4.

back to $\alpha=0$ via pseudoarclength continuation. Figure 4 illustrates this homotopic unfolding in $\alpha$ for a constant value of $R=8$, which corresponds to continuations along the cross-section depicted as a dashed gray vertical line in Fig. 3(b). To guide the eye, color coded bullets have been placed along the gray dashed line in order to identify the unfolded branches. For $R=8$, we have overall identified 7 solution branches: $\mathrm{A}_{1,2,3}, \mathrm{~B}_{1,2}$, and $\widetilde{\mathrm{B}}_{1,2}$. Branches $\mathrm{A}_{i}$ are $H$-symmetric, while branches $\widetilde{\mathrm{B}}_{1}$ and $\widetilde{\mathrm{B}}_{2}$ are in fact $H$-symmetry-related to the $\mathrm{B}_{1}$ and $\mathrm{B}_{2}$, respectively: a reflection $z \rightarrow-z$ plus a reflection around $\alpha=\pi / 4$ (and $5 \pi / 4$ ). Figures 5 and 6 illustrate panels of the velocity profiles $U, V$, and $W$ of each one of the genuinely independent types of flows (i.e., not related through symmetry transformations) for $R=8$ and for certain selected values of $\alpha$, covering representative stretching-shrinking configurations of the top and bottom plates. To avoid redundancy, the panels only include values of $\alpha$ corresponding to genuinely different stretchingshrinking configurations of the plates. For example, the case $\alpha=0$ is not included, since it can be recovered from the case $\alpha=\pi / 2$ (after performing a suitable symmetry transformation), as 


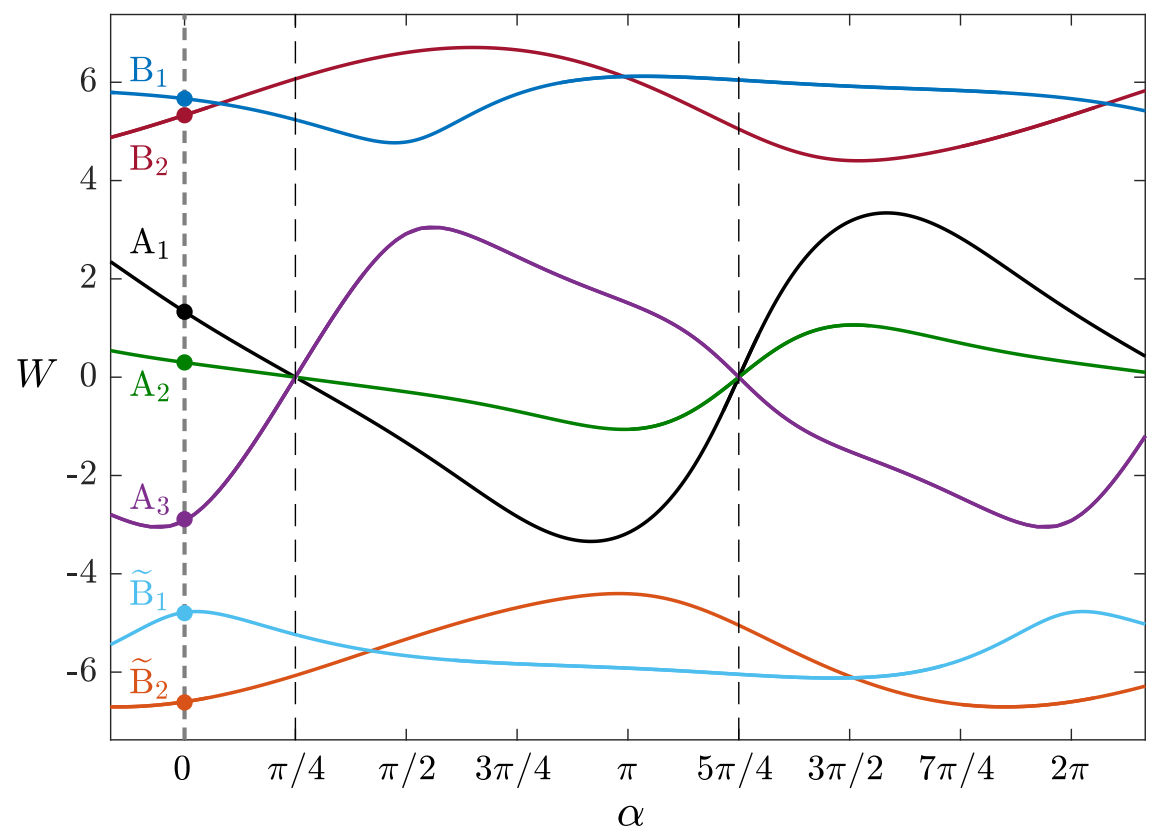

FIG. 4. Wall-normal velocity $W$ at mid plane $z=0$ corresponding to the families of steady solutions found for $R=8$. A dashed gray vertical line has been placed to guide the eye when identifying the unfolded branches shown in Fig. 3(b) by color coded bullets.

illustrated before for the Wang \& Wu case. All seven families of solutions are in general threedimensional. However, branch $\mathrm{A}_{1}$ solutions become two-dimensional for $\alpha=\pi / 2$ (bottom plate stretching in the $x$-direction, top plate stationary) or $\alpha=\pi$ (top plate shrinking in the $y$-direction and bottom plate stationary). This is clearly reflected in the top panel of Fig. 5, where $V=0$ for $\alpha=\pi / 2$, and $U=0$ for $\alpha=\pi$. A careful inspection of the speed profiles shown in panel $\mathrm{A}_{1}$ of Fig. 5 reveals, as it has been stated in Section §II, a remarkable similarity to those corresponding to the Stokes flow (16) previously shown in Fig. 2. In fact, the $A_{1}$ solution branch is actually the nonlinear continuation of the Stokesian flow when, after increasing the Reynolds number, the nonlinearities can no longer be neglected. Furthermore, Fig. 5 and Fig. 6 also reveal that all solution branches except $\mathrm{A}_{1}$ exhibit their maximum and minimum values of velocities $U$ and $V$ inside the channel, significantly far from the walls and independently of the value of $\alpha$. By contrast, the low relevance of the nonlinear term in the case of $\mathrm{A}_{1}$ produces a simpler solution whose velocity profiles peaks are always governed by the walls. These nonlinear effects, already reported in the two-dimensional extensional channel flow Marques et al. (2017), allow disturbances coming from the boundaries that interfere with the velocity field and produce such kind of solutions. 

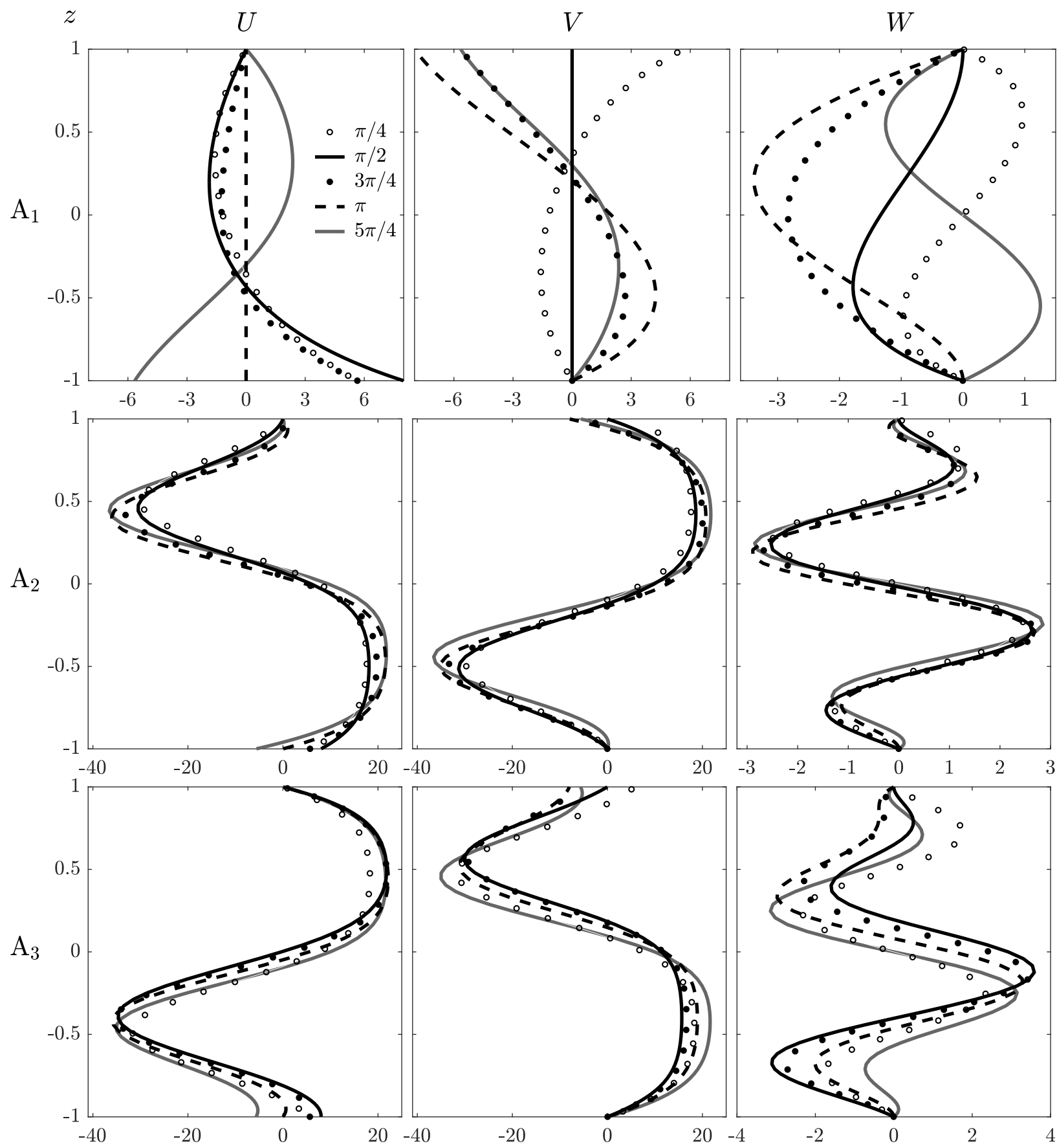

FIG. 5. Structure of the steady solutions $\mathrm{A}_{1}, \mathrm{~A}_{2}$, and $\mathrm{A}_{3}$, at $R=8$ for the $\alpha$ values indicated. Panels show velocity profiles of $U, V$ and $W$.

To have more insight regarding the flow structure of these new families of solutions, and to compare them with the Stokesian base flow, Fig. 7 depicts helicity $h$ isocontours of these solutions over a cylinder of unit radius placed at $(x, y, z)=(0,0,0)$. A visual inspection of $h$ confirms that all 


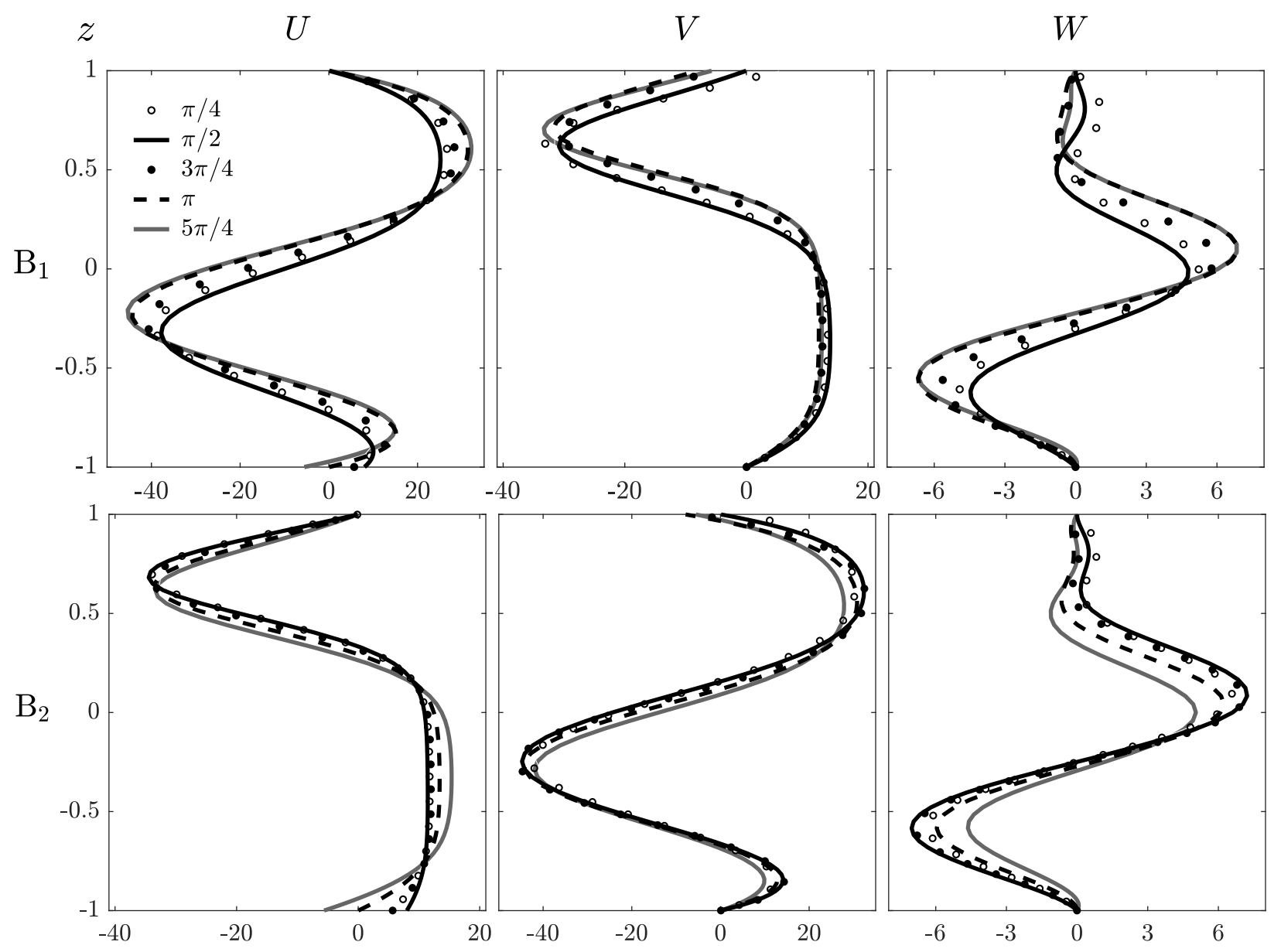

FIG. 6. Same as Fig. 5 for solutions $\mathrm{B}_{1}$ and $\mathrm{B}_{2}$. The profiles of the solutions $\widetilde{\mathrm{B}}_{1,2}$ have not been included, as they are symmetry related to $\mathrm{B}_{1,2}$.

solutions satisfy the symmetry relations (26). It is worth mentioning that for $\alpha=\pi / 4$ both the base Stokesian flow and the $\mathrm{A}_{i}$ solutions are self-symmetric: invariant under the $H$-rotation described in (26b). By contrast, the $\mathrm{B}_{i}$ solutions are not $H$-invariant: the symmetry transforms one branch into the other, $H: \mathrm{B}_{i} \mapsto \widetilde{\mathrm{B}}_{i}$. Similarly, Fig. 8 shows radial velocity $v_{r}$ isocontours for the same cases previously depicted in Fig. 7. All solutions satisfy velocity field symmetries (20) in the same terms as the aforementioned for the helicity. As expected, solutions BF and $\mathrm{A}_{1}$ are very similar, both having their maximum radial velocities located at the boundary planes. By contrast, the other solutions show intense internal return jets (in yellow) in the $x$ and $y$ directions. More remarkably, the maximum velocity of these jets is larger than the stretching velocity at the boundaries. A very similar phenomenon has recently been observed in flows arising in stretching pipes, where dominant axial jets appear in the center line of the pipe, and with velocities considerably larger 

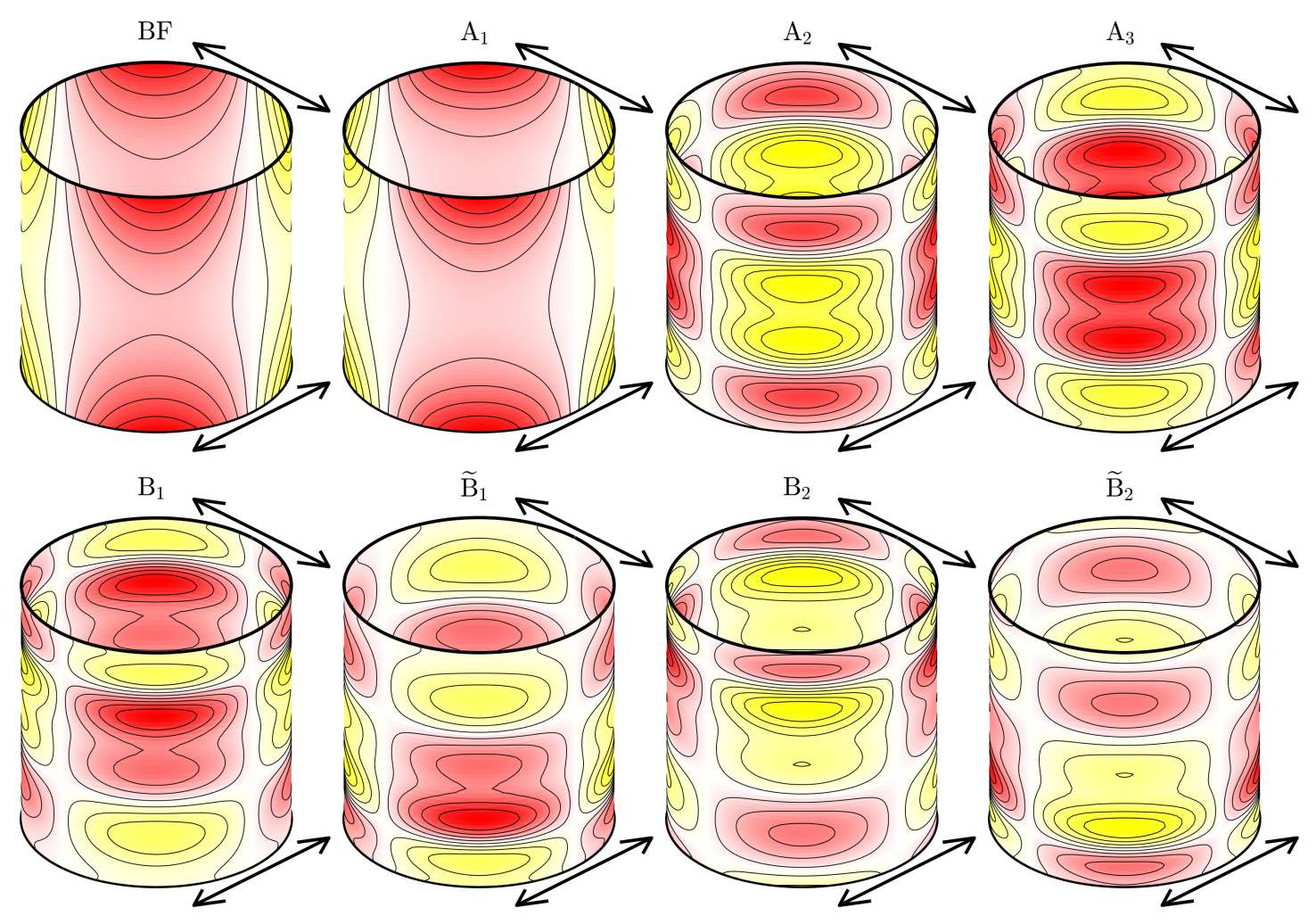

FIG. 7. Isocontours of helicity $h$ over a cylinder of unit radius for base Stokes flow (BF), solutions $A_{1,2,3}$, $\mathrm{B}_{1,2}$ and $\widetilde{\mathrm{B}}_{1,2}$. Red and yellow colors indicate positive and negative $h$ values, respectively. All cases are for $R=8$ and $\alpha=\pi / 4$; top and bottom plates stretching with the same acceleration rate in the directions indicated by the arrows. For a correct interpretation, these projections are aligned with the symmetry plane $x=y$.

than the ones induced nearby the stretching wall Marques and Meseguer (2019).

In order to reveal the origin and fate of these and other solution branches, we have carefully tracked the number of solutions of system (37) throughout the two-dimensional parameter space $(\alpha, R)$. Figure 9(a) summarizes the results by depicting the parameter regions where a different number (boxed) of equilibrium solutions has been identified. These regions are delimited by boundaries (solid black curves) along which solutions are created or annihilated by means of a saddle-node bifurcation (fold) mechanism. The figure has two vertical reflection symmetries, about $\alpha=\pi / 4$ and $5 \pi / 4$ due to the symmetry $H$. The bifurcation curve starting at $C_{1}$ for $\alpha>5 \pi / 4$ has a local minimum at the point $\mathrm{F}_{1}$ (white bullet), located at the coordinates $(\alpha, R)=(4.54,10.54)$. The bifurcation curve symmetric about $\alpha=5 \pi / 4$ has the symmetric of the $\mathrm{F}_{1}$ minimum at $\alpha=3.31$ (not shown). The bifurcation curve starting at $C_{2}$ for $\alpha>\pi / 4$ has its 

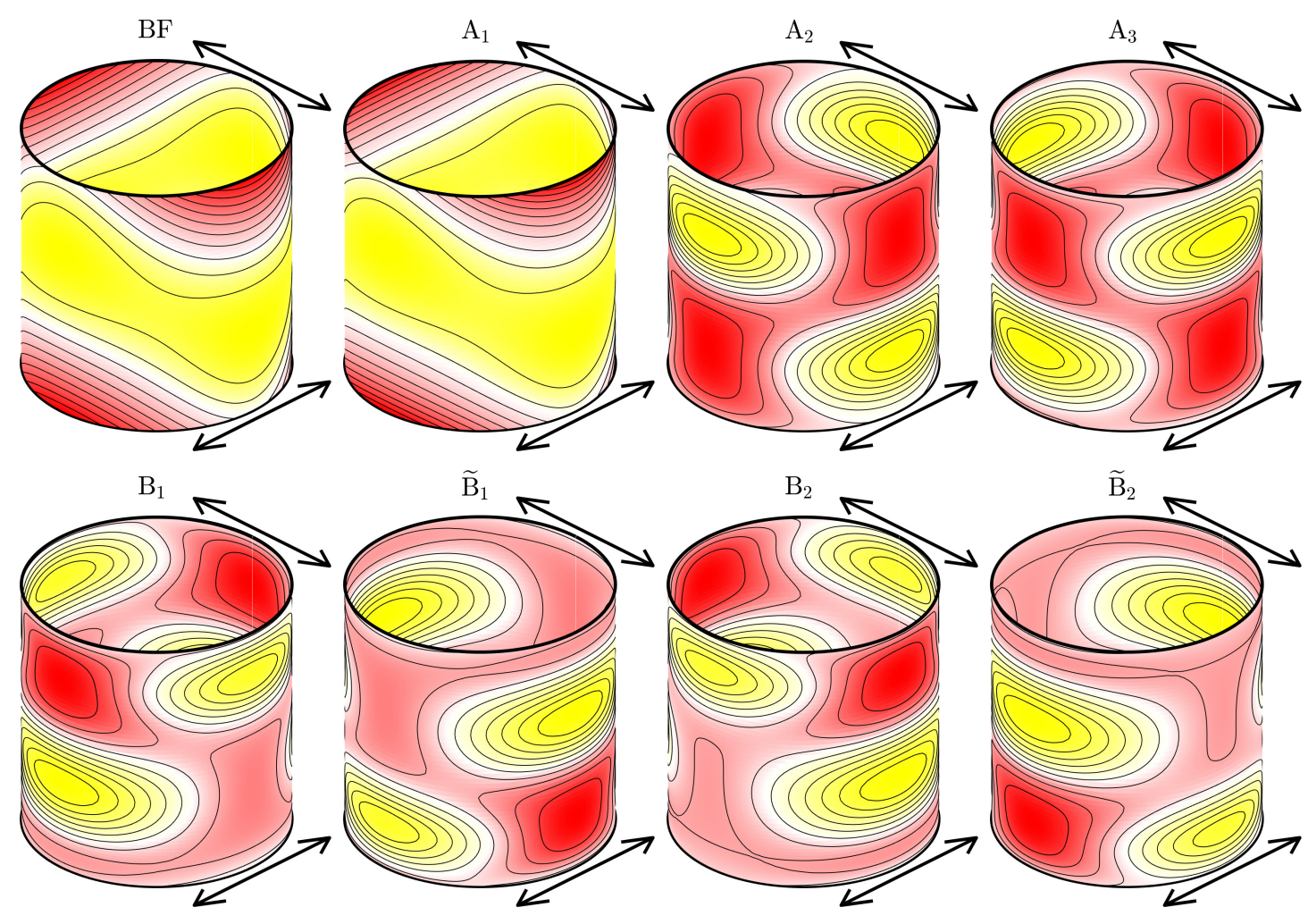

FIG. 8. Isocontours of radial velocity $v_{r}$ for $R=8$ and $\alpha=\pi / 4$. Red and yellow colors indicate positive and negative values of $v_{r}$, respectively.

minimum at the point $F_{2}$, with coordinates $(\alpha, R)=(1.6,9.019)$. The symmetric counterpart with respect $\alpha=\pi / 4$ has the minimum at $\alpha=0.0293$, or $\alpha=6.2539$ due to the natural $2 \pi$-periodicity of the diagram. We have extended the $\alpha$ range beyond the interval $[0.2 \pi]$ in the figure to facilitate following the bifurcaton curves. As a result, the aforementioned starting 7 solutions exist for Reynolds numbers within the range [0,9.019].

For Reynolds numbers slightly below and above the critical value 9.019, and $\alpha$ nearby 0 or $\pi / 2$, two of the seven solutions are lost, as it can be observed from Figures 10(a) and 10(b), for $R=9$ and $R=10$, respectively. Specifically, in a neighbourhood of $\alpha=\pi / 2$ and $R=9.019$, branches $\mathrm{A}_{3}$ and $\mathrm{B}_{1}$ exhibit a pinch, indicated by red vertical arrows in Fig. 10(a) for $R=9$. These two branches eventually merge, annihilating two solutions in that neighbourhood and creating two detached new branches that separate apart, as indicated by red horizontal diverging arrows in Fig. 10 (b) for $R=10$. As expected, $\mathrm{A}_{3}$ curve simultaneously merges with the symmetric $\widetilde{\mathrm{B}}_{1}$ branch for $\alpha=0$ or $\alpha=2 \pi$, also indicated by vertically converging red arrows in Fig. 10(a). For $R=10$, other regions, away from $\alpha=0, \pi / 2,2 \pi$, may still have seven solutions. However, a slight 

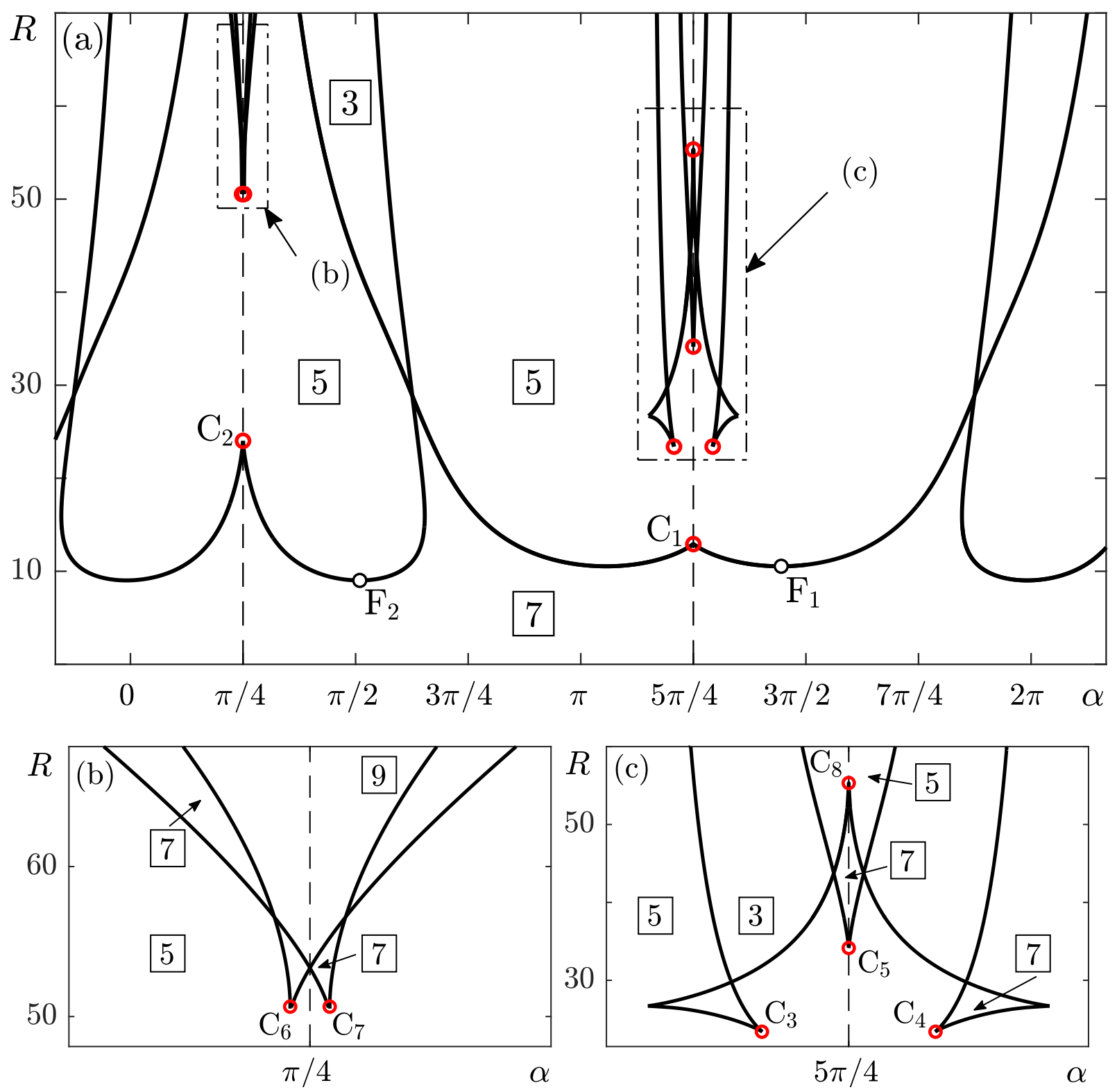

FIG. 9. (a) Explored domain in parameter space $(\alpha, R)$ indicating the number of equilibrium solutions (squared inset numbers) found in each region. (b) - (c) show detailed views of selected regions in (a). Dashed vertical symmetry lines are located at abscissas $\alpha=\pi / 4$ and $\alpha=5 \pi / 4$.

increase of the Reynolds number beyond $R=10.54$ also induces the loss of some of these solutions as well. In particular, branches $\mathrm{A}_{2}$ and $\mathrm{B}_{2}$ exhibit another pinch phenomenon for $R=10.54$ in the neighbourhoods of $\alpha=3 \pi / 2$ (also exhibited by branches $\mathrm{A}_{2}$ and $\widetilde{\mathrm{B}}_{2}$ nearby $\alpha=\pi$ ), as indicated by vertically converging blue arrows in Fig. 10(b). For $R=10.8$, these two pair of branches have already merged and destroyed two solutions nearby $\alpha=3 \pi / 2$ and $\alpha=\pi$, eventually creating two 


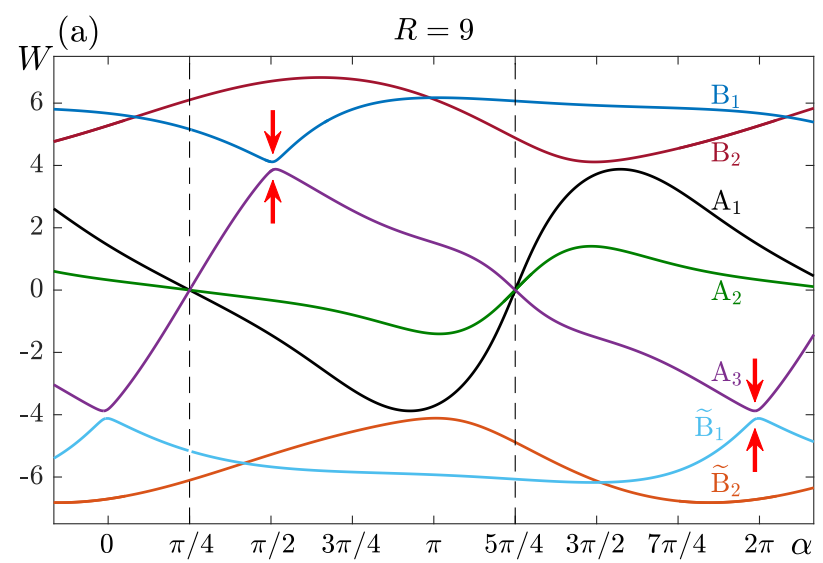

(c)
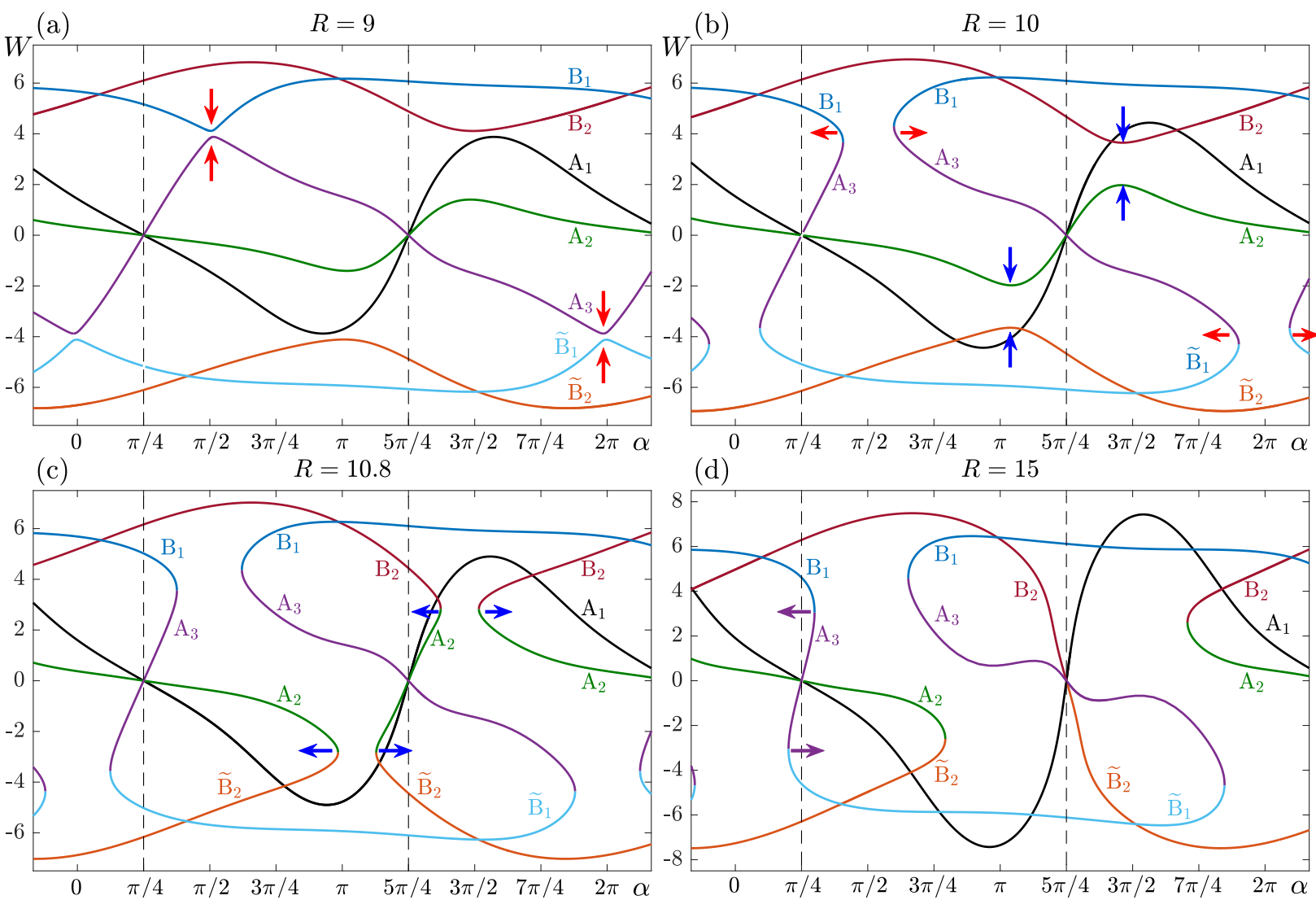

FIG. 10. (a) - (d) Continuations in $\alpha$ of the families of steady solutions for the indicated $R$ values. Red-blue vertically converging and horizontally diverging arrows have been included to indicate the regions where pairs of solutions are lost. Violet arrows in (d) indicate saddle-node bifurcations movements converging to cusp bifurcation point $C_{2}$.

detached independent connections that separate apart, as indicated by horizontal diverging blue arrows in Fig. 10(c). This mechanism of creation-annihilation of branch pairs is similar to the one recently observed in extensional planar or cylindrical flows Marques et al. (2017); Marques and Meseguer (2019).

\section{CODIMENSION-2 CUSP BIFURCATION POINTS}

When crossing the saddle-node bifurcation curves, two solutions merge and disappears. This is the generic situation in steady bifurcations of ODEs or PDEs. However, the presence of the symmetry $H$ (symmetry group $Z_{2}$ ) for $\alpha=\pi / 4$ and $5 \pi / 4$ modify the scenario. The generic steady bifurcation of a $Z_{2}$-symmetric solution $(\alpha=\pi / 4$ or $5 \pi / 4$ fixed, and increasing $R$ ) is a pitchfork. 
Here three different solutions merge and only one survives after the bifurcation; the solution that remains is $Z_{2}$-symmetric, and the other two are symmetrically related Kuznetsov (2004). What we observe in a neighborhood of $\alpha=\pi / 4$ and $5 \pi / 4$ is a cusp bifurcation, of codimension two. The cusp points $\mathrm{C}_{1}, \mathrm{C}_{2}, \mathrm{C}_{5}$ and $\mathrm{C}_{8}$ are of this type, with the symmetry $H$ playing a critical role. Figures $10(\mathrm{c})$ and $10(\mathrm{~d})$ illustrate the formation of the cusp $\mathrm{C}_{1}$, located at $(\alpha, R)=(5 \pi / 4,12.92)$. In Figure $10(\mathrm{c})$, for $\alpha=5 \pi / 4$, we see three solutions $\left(\mathrm{B}_{2}, \mathrm{~A}_{2}\right.$ and $\left.\widetilde{\mathrm{B}}_{2}\right)$ on the same S-shaped bifurcation curve. When increasing $R$ the three solutions approach each other, merge at $R=$ 12.92, and only one remains at larger $R$, as can be seen in Figure 10(d), where the S-shaped curve has become a smooth univalued function. The same happens at cusp $\mathrm{C}_{2}$, located at $(\alpha, R)=$ $(\pi / 4,24.02)$. Figure $10(\mathrm{~d})$ at $R=15$ shows that the three solutions $\mathrm{B}_{1}, \mathrm{~A}_{3}$ and $\widetilde{\mathrm{B}}_{1}$ on the same $\mathrm{S}$-shaped bifurcation curve approach (purple arrows in the figure), and merge at $R=24.02$.

The two saddle-node bifurcation curves that meet at a cusp point are different. For example, at $\mathrm{C}_{1}$, the saddle-node curve that has minimum $\mathrm{F}_{1}$ (for $\alpha>5 \pi / 4$ ) correspond to the merging of $\mathrm{B}_{2}$ and $\mathrm{A}_{2}$, while the symmetric counterpart (for $\alpha<5 \pi / 4$ ) correspond to the merging of $\mathrm{A}_{2}$, and $\widetilde{\mathrm{B}}_{2}$. Two different saddle-node bifurcation curves can meet in the absence of symmetry (away from the lines $\alpha=\pi / 4$ and $5 \pi / 4$ ), resulting also in a codimension-2 cusp bifurcation point without symmetry Kuznetsov (2004). This is what happens at the cusp points $C_{3}, C_{4}, C_{6}$ and $C_{7}$.

For moderately larger Reynolds numbers, our parametric exploration reveals a remarkable increase in the complexity of the topology of equilibria. This feature can be clearly seen in Figures 9(a) and 9(c) for $R=23.4$ and beyond, showing the presence of multiple cuspidal points, namely $\mathrm{C}_{3}$ and $\mathrm{C}_{4}$, for $\alpha=3.792$ and $\alpha=4.062$, very close to their central value $\alpha=5 \pi / 4$, respectively. These two symmetric cuspidal bifurcation points lead to two small regions inside which we may find 7 equilibria again, as summarized in Fig. 9(c). Figures 11(a) and (b) are detailed views slightly below and above $\mathrm{C}_{3,4}$ for $R=22$ and $R=25$, respectively. The simultaneous cusp bifurcations appear along branch $\mathrm{A}_{1}$, bounded by two saddle-node bifurcations (white squares in Fig. 11), leading to the new solution branch A4. As the Reynolds number is increased, Fig. 11(c) shows that $\mathrm{A}_{1}-\mathrm{A}_{4}$ and $\mathrm{A}_{3}$ branches approach and exhibit a pinch near two of the saddle-nodes leading to an $\alpha$-isolated $\mathrm{A}_{1}-\mathrm{A}_{3}$ branch, which is clearly depicted in Fig. 11(d). However, as this isola reduces significantly, two of these seven solutions soon disappear again for $R>30$.

Figure 12 shows the structure of the seven solutions indicated in blue in Fig. 11(d), coexisting for the same value of the parameters $(\alpha, R)=(4.18,27)$, and including two solutions on the isola. These solutions are close to but no equal to $\alpha=5 \pi / 4$, therefore none of them is $H$-symmetric. 

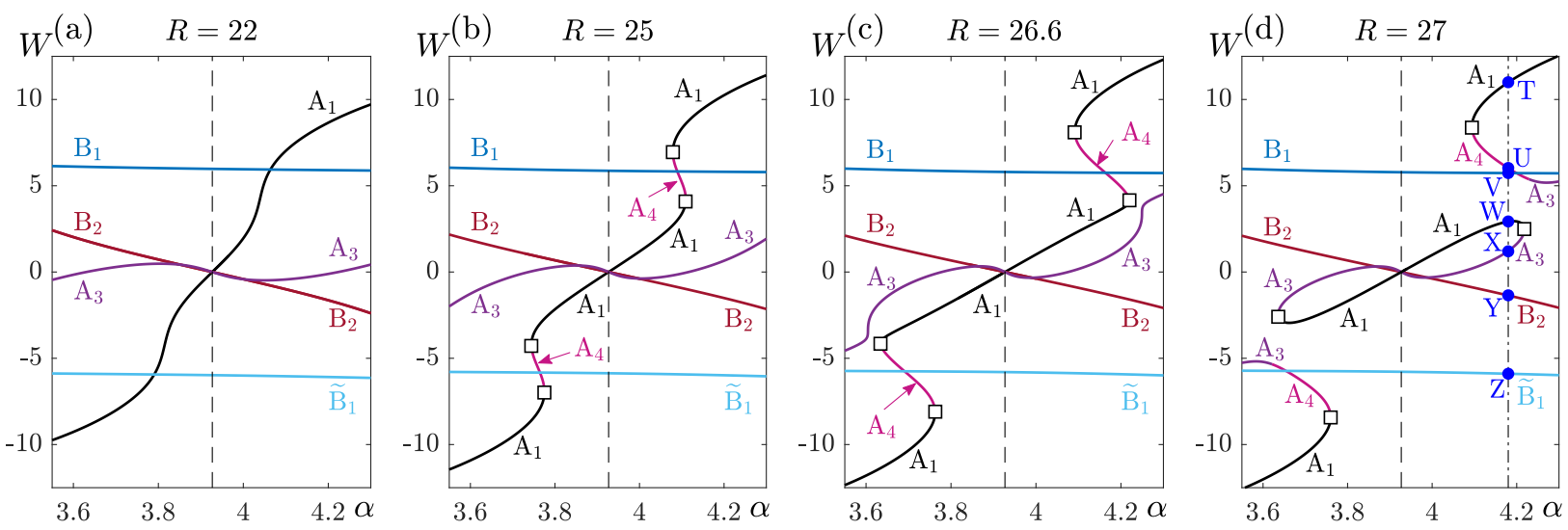

(e) $\quad R=33$

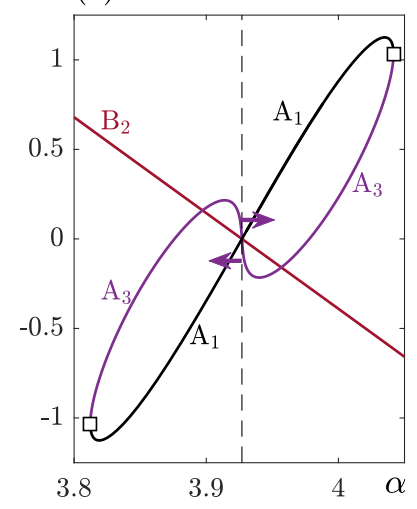

(f) $\quad R=43.73$

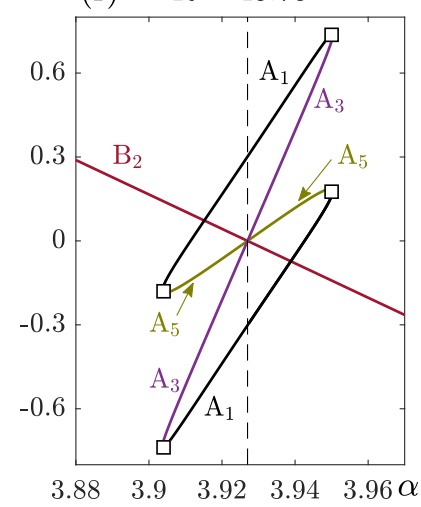

(g) $\quad R=47$

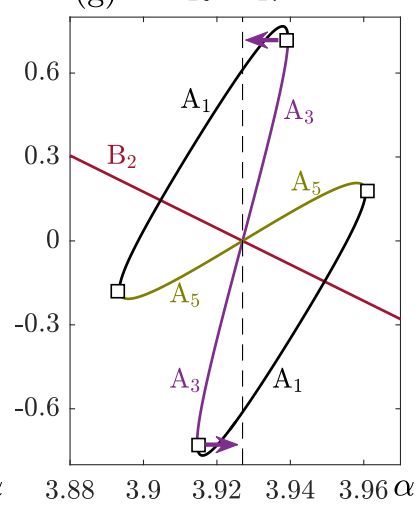

(h) $\quad R=60$

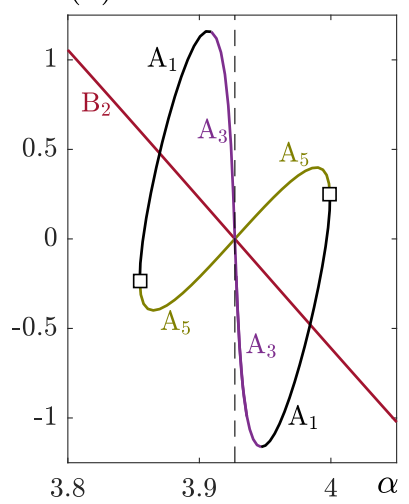

FIG. 11. Continuations in $\alpha$ in a neighbourhood of $\alpha=5 \pi / 4$ (dashed line) for selected Reynolds number. (a) - (d) show all solution branches corresponding to Fig. 9(c). (e) - (h) depict detail continuations of the isolated $A_{1}-A_{3}$ branch. White squares are saddle-node bifurcations and violet arrows indicate branch shifts leading to (e) $\mathrm{C}_{5}$ and (g) $\mathrm{C}_{8}$ cuspidal points for $\alpha=5 \pi / 4$. Blue symbols in (d) correspond to the seven solutions coexisting for the same parameter values $(\alpha, R)=(4.18,27)$ that are described in the text and in Fig. 12.

Comparing these solutions at $R=27$ with the solutions at $R=8$ shown in Fig. 8, there are two main differences. One is the increased strength of the internal return jets. The other difference is that the number of jets increases; this is clearly seen in solutions V and Y, exhibiting four layers, two at the boundary planes, and other two in the interior, with opposite radial velocity. This results in an increased shear stress between layers, which can be a source of dynamical instabilities. This increase in the number of layers and its strength has also been observed in two-dimensional planar channels Marques et al. (2017) when the walls are simultaneously shrinking, as in Figure 12. Notice that the solutions for $R=8$ shown in Fig. 8 correspond to a stretching situation $(\alpha=\pi / 4)$.

For Reynolds numbers within the range $R \in[34.15,55.34]$, and in a very narrow neighbourhood 


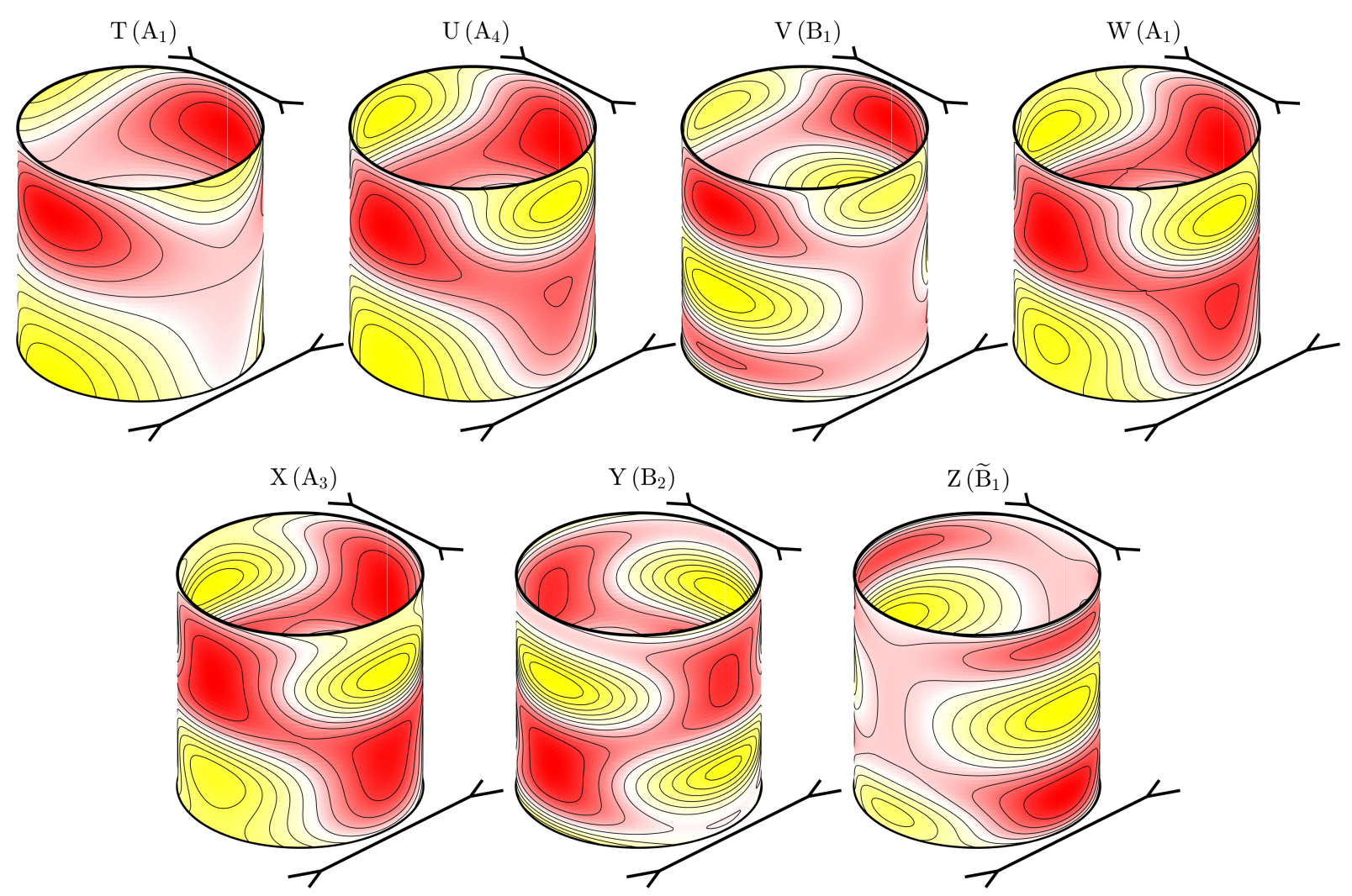

FIG. 12. Radial velocity $v_{r}$ for solutions at $\alpha=4.18$ and $R=27$. Red and yellow colors indicate positive and negative $v_{r}$ values, respectively.

of $\alpha=5 \pi / 4,7$ solutions are available again. This region is bounded by two cusp points, namely $\mathrm{C}_{5}$ and $\mathrm{C}_{8}$, located along the line $\alpha=5 \pi / 4$, for $R=33.73$ and $R=55.34$, respectively. In fact, $\mathrm{C}_{5}$ is the cusp bifurcation located along the $\alpha$-isolated branch responsible for the appearance of a new solution branch, namely $\mathrm{A}_{5}$. Violet arrows in Fig. 11(e) illustrate this process of solution creation by indicating branch displacements and Fig. 11(f) depicts the isola with these new A5 solutions. If the Reynolds number is further increased, the outermost pair of saddle-node bifurcations move towards the symmetry line $\alpha=5 \pi / 4$, indicated with violet arrows in Fig. 11(g), and merge together forming $\mathrm{C}_{8}$. As a result, in a neighbourhood of $\alpha=5 \pi / 4$ and for $R>55.34$ five solutions remain; see Fig. 11(h) for $R=60$. This increase of complexity in the topology of equilibria when the boundaries are shrinking has also been recently reported in flows arising inside contracting pipes and two-dimensional parallel plates Marques et al. (2017); Marques and Meseguer (2019).

We end our analysis by describing the last pair of symmetric cuspidal points that simultaneously appear in a neighbourhood of $\alpha=\pi / 4$ when both plates are stretching at the same higher 

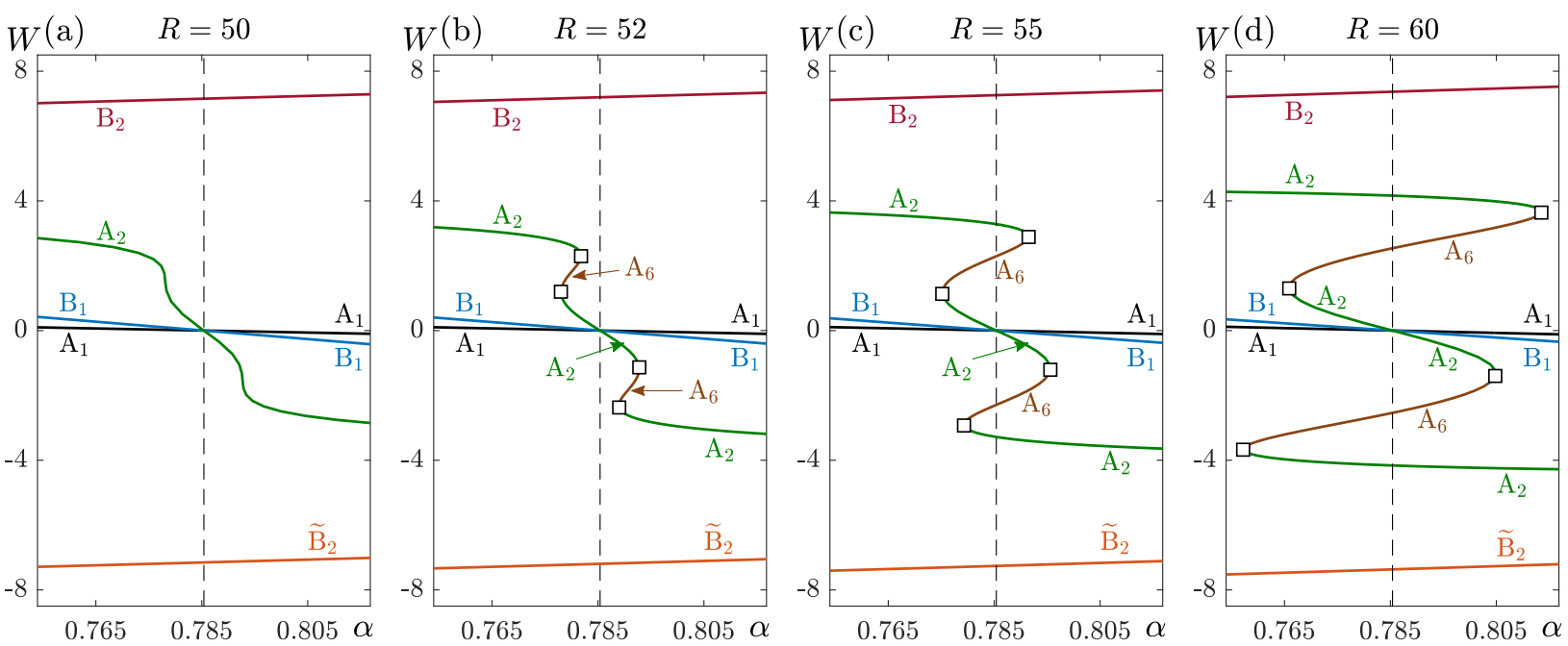

FIG. 13. (a) - (d) Continuations in $\alpha$ in a neighbourhood of $\alpha=\pi / 4$ (dashed line) for selected Reynolds number. White squares indicate saddle-node bifurcations.

accelation rate. Fig. 9(a) depicts these cuspidal points for $R=50.49$, namely $\mathrm{C}_{6}$ and $\mathrm{C}_{7}$, located at $\alpha=0.7784$ and $\alpha=0.7924$, respectively. These two cusps are so close that an inset has been also provided in Fig. 9(b) to facilitate visualization. This pair of symmetric cusps also are responsible for generation of two more solutions in an extremely narrow region of the parameter space. In this case, however, increasing the Reynolds number leads to the creation of a total number of 9 equilibria. For $R \lesssim 50.49$, only 5 solution branches coexist within the interval $\alpha \in[0.755,0.815]$, as shown for example in Fig. 13(a) for $R=50$. For $R \gtrsim 50.49$, after the cuspidal points $\mathrm{C}_{6}$ and $\mathrm{C}_{7}$ appear along branch $\mathrm{A}_{2}$, a new solution branch $\mathrm{A}_{6}$ is born, bounded by two saddle-node points, depicted as white squares in Fig. 13(b) for $R=52$. Further increase of the Reynolds number leads to a separation of these saddle-node points, widening the range of $\alpha$ values for which up to 9 solution branches coexist, as depicted in Fig. 13(c) and Fig. 13(d) in a neighbourhood of $\alpha=\pi / 4$ for $R=55$ and $R=60$, respectively. Table I summarizes the location of the two fold and eight cuspidal points identified in this exploration.

\section{CONCLUSIONS}

A comprehensive exploration of equilibrium flows arising between two parallel plates that stretch-shrink orthogonally and independently has been performed. The exploration has covered a wide range of stretching-shrinking acceleration rates. For moderately low Reynolds numbers, 


\begin{tabular}{|c|c|c|}
\hline Point & $\alpha$ & $R$ \\
\hline $\mathrm{F}_{1}$ & 4.54 & 10.54 \\
\hline $\mathrm{F}_{2}$ & 1.6 & 9.019 \\
\hline $\mathrm{C}_{1}$ & $5 \pi / 4$ & 12.92 \\
\hline $\mathrm{C}_{2}$ & $\pi / 4$ & 24.02 \\
\hline $\mathrm{C}_{3}$ & 3.792 & 23.40 \\
\hline $\mathrm{C}_{4}$ & 4.062 & 23.40 \\
\hline $\mathrm{C}_{5}$ & $5 \pi / 4$ & 33.73 \\
\hline $\mathrm{C}_{6}$ & 0.7784 & 50.49 \\
\hline $\mathrm{C}_{7}$ & 0.7924 & 50.49 \\
\hline $\mathrm{C}_{8}$ & $5 \pi / 4$ & 55.34 \\
\hline
\end{tabular}

TABLE I. Locations of fold and cuspidal points in Fig. 9.

up to seven steady flows have been identified. Three of these flows are genuinely independent, whereas two of the remaining four flows are related through rotational symmetry transformations. For higher Reynolds numbers, however, the number of equilibrium solutions may range between three and nine. For moderate Reynolds numbers, our exploration reveals that equilibria tend to be created or annihilated more likely when both plates are simultaneously shrinking. These new solutions are the result of codimension-2 cusp bifurcations. Similar cuspidal points have been identified for higher Reynolds numbers when both plates are simultaneously stretching. In this case, the cusps only favour the creation of new solutions. All cusp bifurcations and new solutions are close to the $H$-symmetry lines, corresponding to stretching or shrinking planes with the same acceleration rate. In fact it is the breaking of the $H$-rotational symmetry that results in this plethora of new solutions and cusp bifurcations.

The presence of cuspidal points when the boundaries are shrinking is consistent with recent analyses carried out in cylindrical geometries and in two-dimensional channels Marques et al. (2017); Marques and Meseguer (2019). The natural continuation of the present work would be the exploration of the dynamical properties of the found solutions. This would require performing time integration of the original equations in order to clarify the linear and nonlinear stability of these solutions. However, these aspects are far from the scope of the present analysis. 
Orthogonally stretching parallel plates

\section{ACKNOWLEDGMENTS}

This work was supported by the Spanish MINECO Grants No. FIS2017-85794-P and No. PRX18/00179, and the Generalitat de Catalunya Grant No. 2017-SGR-785.

\section{DATA AVAILABILITY}

The data that support the findings of this study are available from the corresponding author upon reasonable request.

\section{Appendix A: Spectral convergence}

All the solutions reported in the present study have been confirmed to satisfy spectral convergence, that is, the exponential decay of the coefficients $a_{m}$ and $b_{m}$ corresponding to the Legendre expansions (34). Figure 14 illustrates this property by depicting on a linear-log plot the modulus of these coefficients as function of $m$. Figure 14(a) shows the decay of the Legendre coefficients corresponding to the 2-dimensional solution of (Wang and Wu, 1995) shown in Fig. 3(a) for $R=90$ and $\alpha=0$. In this case, the lower plate is at rest, whereas the upper one is stretching at the maximum acceleration rate, inducing fluid motion in the $y$ direction only, the $u$-component of the velocity therefore being zero. This is reflected in the almost negligible, to machine precision, magnitude of the $a_{m}$ Legendre coefficients associated with the $u$-component of the flow. The $v$ component of the flow is governed by the $b_{m}$ coefficients, that show a clear exponential decay, covering nearly 14 orders of magnitude, as a distinctive characteristic of spectral convergence. Figure 14(b) corresponds to the Legendre coefficients of the solution $\mathrm{Z}\left(\widetilde{\mathrm{B}_{1}}\right)$ shown in Fig. 12, for $R=27$. In this case, the fluid motion has non-zero components in the $x$ and $y$ directions and exhibits multiple inner jets. We have chosen this solution precisely because of its complexity, therefore demanding a higher number of Legendre modes. In this case, both coefficients also exhibit a very neat spectral convergence law, overall showing an exponential decay of early 12 orders of magnitude using $M=50$ Legendre polynomials.

The reliability of the Legendre spectral discretization used in this work has been tested by computing the coordinates of cuspidal and fold points, and for a number of Legendre polynomials within the range $M \in[20,55]$. Table II outlines this test, exhibiting an outstanding exponential convergence in the coordinates of the codimension- 1 and 2 bifurcation points $\mathrm{F}_{2}$ and $\mathrm{C}_{8}$, respectively, 
Orthogonally stretching parallel plates

(a)

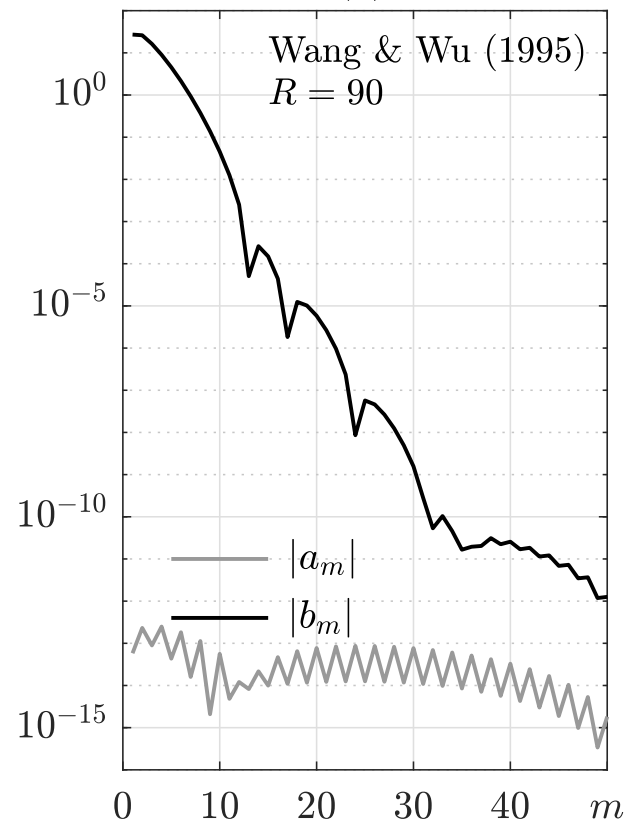

(b)

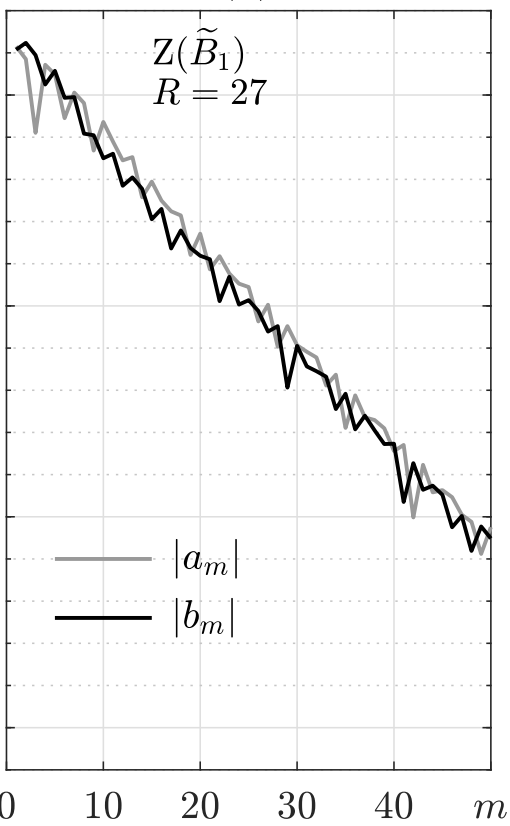

FIG. 14. Exponential convergence of the spectral Legendre expansions (34).

\begin{tabular}{|c|c|c|c|}
\hline$M$ & $R\left(\mathrm{~F}_{2}\right)$ & $\alpha\left(\mathrm{F}_{2}\right)$ & $R\left(\mathrm{C}_{8}\right)$ \\
\hline 20 & 9.0189805916 & 1.5981603583 & 55.3506994615 \\
\hline 30 & 9.0190144077 & 1.5981489100 & 55.3359422714 \\
\hline 40 & 9.0190143788 & 1.5981488209 & 55.3359568841 \\
\hline 45 & 9.0190143803 & 1.5981488213 & 55.3359562436 \\
\hline 50 & 9.0190143793 & 1.5981488215 & 55.3359567273 \\
\hline 55 & 9.0190143793 & 1.5981488213 & 55.3359561122 \\
\hline
\end{tabular}

TABLE II. Locations of fold $\mathrm{F}_{2}$ and cusp $\mathrm{C}_{8}$ points in the $(\alpha, R)$-plane as a function of the Legendre modes, $M$, used in the discretization. The cuspidal point appears along the symmetry line $\alpha=5 \pi / 4$.

shown in Fig.9. We can conclude that $M=50$ Legendre polynomials already provides nearly 10 exact figures in the critical $R$ of the fold point, and 6 in the cuspidal one (located at $\alpha=5 \pi / 4$ ). Increasing the number of Legendre polynomials does not improve the accuracy of the presented results, being overall more than satisfactory. 
Orthogonally stretching parallel plates

\section{REFERENCES}

Ayats, R., Deguchi, K., Mellibovsky, F., and Meseguer, A., "Fully nonlinear mode competition in magnetised Taylor-Couette flow,” J. Fluid Mech. 897, A14 (2020).

Ayats, R., Meseguer, A., and Mellibovsky, F., "Symmetry-breaking waves and space-time modulation mechanisms in two-dimensional plane Poiseuille flow," Physical Review Fluids 5, 094401 (2020).

Brady, J. F. and Acrivos, A., "Steady flow in a channel or tube with an accelerating surface velocity. An exact solution to the Navier-Stokes equations with reverse flow," J. Fluid Mech. 112, 127150 (1981).

Crane, L. J., "Flow past a stretching plate," Zeitschrift für Angewandte Mathematik und Physik (ZAMP) 21, 645-647 (1970).

Deguchi, K., Meseguer, A., and Mellibovsky, F., "Subcritical equilibria in Taylor-Couette flow," Phys. Rev. Lett. 112, 184502 (2014).

Drazin, P. G. and Riley, N., The Navier-Stokes Equations, A Classification of Flows and Exacts Solutions (Cambridge University Press, Cambridge, 2006).

Espín, L. and Papageorgiou, D. T., "Flow in a channel with accelerating or decelerating wall velocity: A comparison between self-similar solutions and Navier-Stokes computations in finite domains," Phys. Fluids 21, 113601 (2009).

Kelley, C., Solving nonlinear equations with Newton's method (SIAM, 2003).

Kuznetsov, Y. A., Elements of Applied Bifurcation Theory, 3rd ed. (Springer, 2004).

Marques, F. and Meseguer, A., "Extensional and torsional pipe flow," Physical Review Fluids 4, 103908 (2019).

Marques, F., Meseguer, A., Mellibovsky, F., and Weidman, P., "Extensional channel flow revisited: a dynamical systems perspective,” Proc. Roy. Soc. Lond. A 473, 20170151 (2017).

Meseguer, A., Fundamentals of Numerical Mathematics for Physicists and Engineers (John Wiley \& Sons, 2020).

Meseguer, A., Mellibovsky, F., Avila, M., and Marques, F., "Families of subcritical spirals in highly counter-rotating Taylor-Couette flow,” Phys. Rev. E 79, 036309 (2009).

Quarteroni, A., Sacco, R., and Saleri, F., Numerical mathematics, Vol. 37 (Springer Science \& Business Media, 2010).

Wang, C.-A. and Wu, T.-C., "Similarity solutions of steady flows in a channel with accelerating 
Orthogonally stretching parallel plates

walls," Comput. Math. Applic. 30, 1-16 (1995).

Wang, C. Y., "The three-dimensional flow due to a stretching flat surface," Phys. Fluids 27, 1915 (1984).

Wang, C. Y., "Review of similarity stretching exact solutions of the navier-stokes equations," Eur. J. Mechanics - B/Fluids 30, 475-479 (2011).

Waters, S. L., "Solute uptake through the walls of a pulsating channel," J. Fluid Mech. 433, 193$208(2001)$.

Waters, S. L., "A mathematical model for the laser treatment of heart disease," J. Biomech. 37, 281-288 (2004).

Weidman, P. and Ishak, A., "Multiple solutions of two-dimensional and three-dimensional flows induced by a stretching flat surface," Communications in Nonlinear Science and Numerical Simulation 25, 1-9 (2015). 